\title{
Evidence for a coupled magnetic-crystallographic transition in $\mathrm{La}_{0.9} \mathrm{Ce}_{0.1} \mathrm{Fe}_{12} \mathrm{~B}_{6}$
}

\author{
L. V. B. Diop $\odot,,^{1, *}$ T. Faske, ${ }^{2}$ M. Amara, ${ }^{3}$ D. Koch $\odot,{ }^{2}$ O. Isnard $\odot,{ }^{3}$ and W. Donner $\odot^{2}$ \\ ${ }^{1}$ Université de Lorraine, CNRS, IJL, F-54000 Nancy, France \\ ${ }^{2}$ Institute of Materials Science, Technical University of Darmstadt, D-64287 Darmstadt, Germany \\ ${ }^{3}$ Université Grenoble Alpes, CNRS, Institut NEEL, 25 rue des martyrs, F-38042 Grenoble, France
}

(Received 8 May 2021; revised 22 August 2021; accepted 30 September 2021; published 14 October 2021)

\begin{abstract}
Competition between antiferromagnetic (AFM), paramagnetic (PM), and ferromagnetic (FM) states in $\mathrm{La}_{0.9} \mathrm{Ce}_{0.1} \mathrm{Fe}_{12} \mathrm{~B}_{6}$ compound is investigated by means of temperature- and magnetic field-dependent $\mathrm{x}$-ray diffraction, magnetization, linear thermal expansion, and magnetostriction experiments. It is shown that both AFM and PM phases get converted into the FM phase via a first-order metamagnetic transition, which is accompanied by a huge forced-volume magnetostriction $\Delta V / V(25 \mathrm{~K}, 6 \mathrm{~T})=1.15 \%$. X-ray powder diffraction reveals a magnetic field-induced crystallographic phase transition from a $R \overline{3} m$ rhombohedral (AFM, PM) to a $C 2 / m$ monoclinic (FM) structure. A peculiarly anisotropic lattice expansion as well as giant negative thermal expansion with a volumetric thermal expansion coefficient $\alpha_{V}=-193 \times 10^{-6} \mathrm{~K}^{-1}$ are observed. These findings point to the significance of magnetoelastic effects in this metamagnet and illustrate the strength of the coupling between lattice and spin degrees of freedom in the $\mathrm{La}_{0.9} \mathrm{Ce}_{0.1} \mathrm{Fe}_{12} \mathrm{~B}_{6}$ intermetallic compound.
\end{abstract}

DOI: 10.1103/PhysRevB.104.134412

\section{INTRODUCTION}

A large number of solid systems display structural phase transitions when subject to changes in external applied pressure, temperature, and chemical composition. However, the occurrence of magnetic field-induced structure transformations is rather exceptional and a few examples are reported in the literature. An intensively investigated case is the transition-metal chalcogenide MnAs which exhibits a first-order structural transition from a hexagonal to an orthorhombic crystal symmetry upon heating through $\approx 313 \mathrm{~K}$ (ferromagnetic ordering temperature) [1-3]. The structural transition observed in this compound can be stimulated, at a fixed temperature, by the application of an external magnetic field. For example at $327 \mathrm{~K}$, the magnetic field required to accomplish the structural phase transformation amounts to $5.1 \mathrm{~T}(3.1 \mathrm{~T})$ for increasing (decreasing) applied fields [1-3]. A work by Asamitsu et al. [4], including striction, $\mathrm{x}$-ray powder diffraction, and resistivity studies as a function of both external field and temperature, has revealed the existence of a magnetic field-induced structural phase transition in the $(\mathrm{La}, \mathrm{Sr}) \mathrm{MnO}_{3}$ series of magnetoresistive compounds. The $\mathrm{Gd}_{5} \mathrm{Ge}_{4}$ alloy has been widely investigated due to a combination of intriguing physical phenomena and potentially important functionalities, such as magnetoresistance, anisotropic magnetostrictive effect, unconventional glasslike magnetic behavior, and unusual kinetic arrest of the magnetostructural phase transformation [5-7]. The temperature alone cannot trigger the ferromagnetic ordering in $\mathrm{Gd}_{5} \mathrm{Ge}_{4}$ intermetallic system, yet a magnetic field induces the ferromagnetic phase through a first-order transition that is coupled with a considerable reshuffling of the crystallographic structure of the compound [8]. The pseudobinary

*leopold.diop@univ-lorraine.fr
$\mathrm{Gd}_{5}\left(\mathrm{Si}_{x} \mathrm{Ge}_{1-x}\right)_{4}$ alloys within the concentration range $0.24 \leqslant$ $x \leqslant 0.5$ are of particular interest; the crystallographic symmetry is no longer orthorhombic but monoclinic, giving rise to a dramatic change in the magnetic behavior $[9,10]$. These compounds undergo a field-induced magnetostructural transition from a high-temperature monoclinic (paramagnetic) to a low-temperature orthorhombic (ferromagnetic) phase, which is accompanied by large magnetoelastic and giant magnetocaloric effects $[10,11]$. Other systems, such as doped-CeFe 2 [12], ( $\mathrm{Hf}, \mathrm{Nb}) \mathrm{Fe}_{2}$ [13], and $\mathrm{FeRh}$ [14-16] were found to exhibit a first-order magnetostructural phase transition from antiferromagnetic to ferromagnetic order. Currently, these different classes of materials featuring a strong coupling between the magnetic and crystal lattices are of significant importance from the fundamental research side as well as from the technological applications viewpoint. The understanding of the interplay between magnetic and crystallographic structures is a crucial challenge in condensed-matter physics. Solid systems with interconnected magnetic and structural degrees of freedom often present multifunctional properties such as giant magnetoresistance, colossal magnetostriction, and giant magnetocaloric effect. These prominent magnetoresponsive effects result from instabilities in crystallographic lattice and magnetic order [9]. In other words, these emergent physical phenomena are particularly pronounced in the vicinity of a magnetostructural phase transformation, which in turn allows controlling the physical properties of the compound via several types of externally applied driving forces. These compounds constitute an exceptional playground for materials physics owing to the extreme sensitivity of their physical properties to moderate external stimuli.

Interestingly, unconventional multistep metamagnetic transitions were recently reported in $(\mathrm{La}, \mathrm{Ce}) \mathrm{Fe}_{12} \mathrm{~B}_{6}$ series of compounds [17-20]. These peculiar metamagnetic phase transitions are featured by ultrasharp steps followed by plateaus leading to an unusual and even unique staircaselike 
magnetization process. The antiferromagnetic itinerantelectron compound $\mathrm{LaFe}_{12} \mathrm{~B}_{6}$ occupies a special place among rare-earth iron-rich intermetallics; it exhibits exotic magnetic and physical properties. The unusual amplitude-modulated spin configuration defined by a propagation vector $\mathbf{k}=$ $(1 / 4,1 / 4,1 / 4)$, remarkably weak Fe magnetic moment $\left(0.43 \mu_{\mathrm{B}}\right)$ in the antiferromagnetic ground state, especially low magnetic ordering temperature $T_{\mathrm{N}}=36 \mathrm{~K}$ for an Fe-rich phase, a multicritical point in the complex magnetic phase diagram [17], both normal and inverse magnetocaloric effects [21], colossal spontaneous magnetization jumps [19], and huge hydrostatic pressure effects [22] can be highlighted as the most relevant. These singular properties not only stimulate the development of theoretical models and experiments under extreme conditions for a deeper understanding of the striking phenomenology of this magnetic system [22-26], but also emphasize the potential interest of $\mathrm{LaFe}_{12} \mathrm{~B}_{6}$ material for future low-temperature energy technologies. Among the $R T_{12} \mathrm{~B}_{6}$ family (where $R$ stands for a rare-earth atom and $T$ is a $3 d$ transition-metal element), $\mathrm{LaFe}_{12} \mathrm{~B}_{6}$ is the sole stable Fe-based phase of the 1:12:6 ternary system $[25,26]$. The first Fe-based member of the $R T_{12} \mathrm{~B}_{6}$ family to be identified, $\mathrm{NdFe}_{12} \mathrm{~B}_{6}$ is metastable [27]. On the other hand, the $R \mathrm{Co}_{12} \mathrm{~B}_{6}$ alloys are stable along the entire rare-earth series [28]. At room temperature, the intermetallic compounds $R T_{12} \mathrm{~B}_{6}$ crystallize in the rhombohedral $\mathrm{SrNi}_{12} \mathrm{~B}_{6}$-type structure (space group $R \overline{3} m$ ) [29-31]. Within the unit cell, there are two crystallographically inequivalent sites for $T$ atoms (18g and $18 h$ ). The $R$ and B atoms occupy the $3 a$ and $18 h$ Wyckoff positions, respectively. The $\mathrm{LaFe}_{12} \mathrm{~B}_{6}$ compound is unique among the ternary system $R T_{12} \mathrm{~B}_{6}$ in having an antiferromagnetic ground state. The magnetic transition temperature of $\mathrm{LaFe}_{12} \mathrm{~B}_{6}$ is much smaller than the Curie point of the Cobased $R \mathrm{Co}_{12} \mathrm{~B}_{6}$ ferro- $(R=\mathrm{Y}, \mathrm{La}-\mathrm{Sm})$ or ferri- $(R=\mathrm{Gd}-$ $\mathrm{Tm})$ magnets $\left(T_{\mathrm{C}}=134-162 \mathrm{~K}\right)$ [28] and an order of magnitude smaller compared to the magnetic ordering temperature of any rare-earth iron-rich binary intermetallic. Extraordinary magnetotransport effects have been most recently discovered in $R T_{12} \mathrm{~B}_{6}$ compounds [32].

In this paper we report a thorough experimental study of the low-temperature first-order phase transition in $\mathrm{La}_{0.9} \mathrm{Ce}_{0.1} \mathrm{Fe}_{12} \mathrm{~B}_{6}$ model compound by combining magnetization, linear thermal expansion, magnetostriction, and temperature- and magnetic field-dependent $\mathrm{x}$-ray powder diffraction measurements. It is thus shown that the metamagnetic transition in question takes place simultaneously with a change of the crystal symmetry: a first-order structural transformation to a different monoclinic phase. This coupled magnetic-crystallographic phase transition is triggered by the external applied magnetic field, resulting in large magnetoelastic effects and therefore making this compound a potential candidate for magnetostrictive materials.

\section{EXPERIMENT DETAILS}

The polycrystalline alloy of composition $\mathrm{La}_{0.9} \mathrm{Ce}_{0.1} \mathrm{Fe}_{12} \mathrm{~B}_{6}$ was prepared by arc melting the mixture of high-purity components (better than 99.9\%) under an atmosphere of argon. To purify the argon atmosphere inside the arc melter, a piece of titanium was melted in an adjacent recess of the water-cooled copper hearth prior to the melting of the constituting elements. To promote compositional homogeneity, the alloy was melted several times with the button being flipped over after each remelting. The so-obtained ingot was wrapped in tantalum foil, sealed in an evacuated fused silica tube, and subsequently annealed in a furnace as follows. It was heated up to $1173 \mathrm{~K}$ at a rate of $5 \mathrm{~K} / \mathrm{min}$ and kept at this temperature for 3 weeks. The analysis of the phase purity and the room-temperature crystallographic structure was carried out by standard x-ray diffraction using a Siemens D5000 powder diffractometer in reflection mode with the Bragg-Brentano geometry and Co$\mathrm{K} \alpha$ radiation $\left(\lambda_{\mathrm{K} \alpha 1}=1.78897 \AA\right.$ and $\left.\lambda_{\mathrm{K} \alpha 2}=1.79285 \AA\right)$.

Magnetization curves were collected on a powder sample at temperatures ranging between 2 and $300 \mathrm{~K}$ with an extraction-type magnetometer. Temperature and field dependences of the magnetic moment were recorded in static magnetic fields of up to $10.5 \mathrm{~T}$. The magnetometer was described in Ref. [33]. Magnetization values were corrected for the presence of the minor ferromagnetic $\mathrm{Fe}_{2} \mathrm{~B}$ secondary phase.

Linear thermal expansion (LTE) and forced magnetostriction measurements were undertaken on the magnetostriction setup of the Néel Institut. This apparatus is based on a highsensitivity capacitance dilatometer that operates in a magnetic field up to $6 \mathrm{~T}$ and over a temperature interval from 2.5 to $300 \mathrm{~K}$. In terms of relative length variation, the sensitivity is better than $10^{-7}$. The capacitance cell can be rotated around the vertical axis of the cryostat while the external magnetic field is applied along a fixed horizontal direction. Magnetostriction isotherms were recorded in both directions parallel and perpendicular to the applied magnetic field. For LTE and magnetostriction experiments, the polycrystal was first subject to mechanical shaping (roughly shaped into sphere) by grinding off sharp corners. Thereupon, it was run for several hours by a jet of compressed air inside a cylinder lined with fine sandpaper, until it became visually spherical. The polycrystalline sample was approximately a sphere of about $5 \mathrm{~mm}$ diameter. The LTE data were corrected for the cell thermal hysteresis.

Temperature- and magnetic field-dependent $\mathrm{x}$-ray powder diffraction data were collected on a custom-built diffractometer in transmission geometry using Mo-K $\alpha$ radiation $\left(\lambda_{\mathrm{K} \alpha 1}=\right.$ $0.70932 \AA$ and $\lambda_{\mathrm{K} \alpha 2}=0.71340 \AA$ ) with an angular $2 \theta$ range from $7^{\circ}$ to $57^{\circ}$ and a scan step of $0.009^{\circ}$. $\mathrm{La}_{0.9} \mathrm{Ce}_{0.1} \mathrm{Fe}_{12} \mathrm{~B}_{6}$ fine powder (particle size smaller than $20 \mu \mathrm{m}$ ) was uniformly mixed with a NIST standard reference Si powder 640d and then glued onto a carbon foil. The sample was dried in air. The carbon foil was mounted on a copper cold finger-serving as a sample holder-of a closed-cycle helium cryofurnace and transferred into a split-coil superconducting magnet that produces a homogeneous magnetic field of up to $5.5 \mathrm{~T}$ around the sample position with the magnetic field vector perpendicular to the scattering plane. A detailed description of the laboratory-based x-ray powder diffractometer can be found in Ref. [34]. Zero-field cooled warming (ZFCW) and field cooled cooling (FCC) experimental procedures were employed for thermodiffraction measurements under various applied magnetic fields (isofield measurements). For ZFCW protocol, the sample is first cooled in zero magnetic field from room temperature down to the lowest measurement 


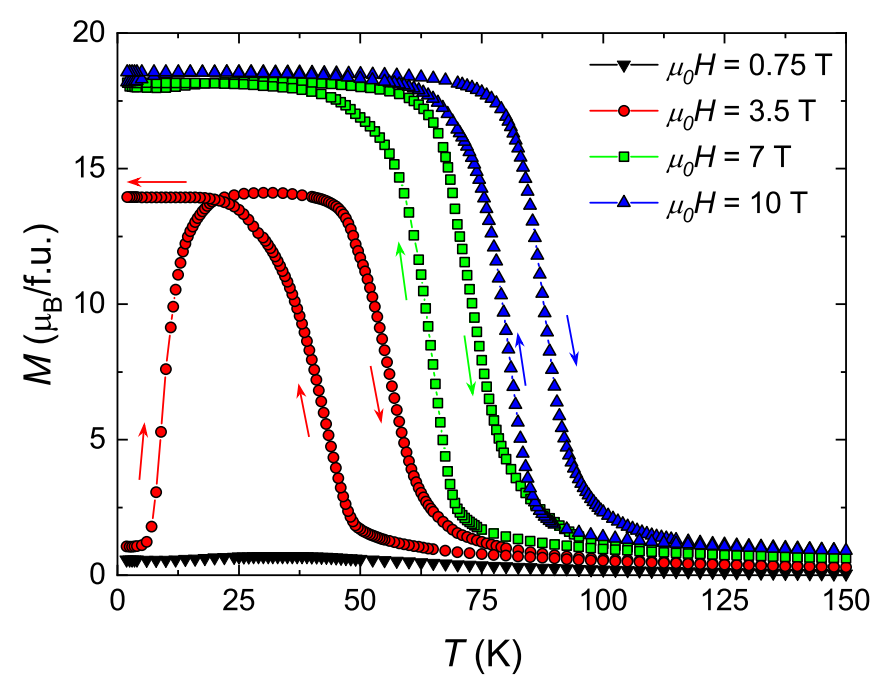

FIG. 1. Thermomagnetic curves of $\mathrm{La}_{0.9} \mathrm{Ce}_{0.1} \mathrm{Fe}_{12} \mathrm{~B}_{6}$ in magnetic fields of $0.75,3.5,7$, and $10 \mathrm{~T}$. Both ZFCW and FCC data are marked by the same symbols. The arrows indicate the direction of the temperature change.

temperature; then, the magnetic field is applied after reaching thermal equilibrium, and diffraction patterns are recorded upon heating. The ZFCW data collection is immediately followed by cooling under the same applied magnetic field (FCC). Prior to the magnetic field-dependent diffraction measurements (isothermal measurements), the sample was cooled from room temperature to the temperature of the measurement with no magnetic field applied. For both isofield and isothermal measurements, the temperature of the sample was stabilized for $\approx 10 \mathrm{~min}$ before diffraction data acquisition. Rietveld analyses of the diffraction patterns were carried out using the FULLPROF suite software package [35]. The unit-cell parameters and phase quantities, when two distinct crystallographic phases coexist in certain combinations of magnetic field and temperature, were determined. The sample employed in the present study is the same as that used in Ref. [20].

\section{RESULTS AND DISCUSSION}

\section{A. Magnetization}

Figure 1 illustrates the thermomagnetic curves, $M(T)$, of $\mathrm{La}_{0.9} \mathrm{Ce}_{0.1} \mathrm{Fe}_{12} \mathrm{~B}_{6}$ in $\mathrm{ZFCW}$ and $\mathrm{FCC}$ measuring protocols at different applied magnetic fields from 0.75 to $10 \mathrm{~T}$. The lowfield magnetization curve $\left(\mu_{0} H=0.75 \mathrm{~T}\right)$ displays a small broad peak around the Néel temperature $T_{\mathrm{N}}=35 \mathrm{~K}$ which corresponds to an antiferromagnetic (AFM)-paramagnetic (PM) phase transition. In magnetic fields of $\mu_{0} H=7$ and $10 \mathrm{~T}$ at $2 \mathrm{~K}$ the alloy is already in the ferromagnetic (FM) state, and therefore the $M(T)$ measurements reflect only the magnetic transition from FM order to PM state.

However, the magnetization shows considerably different thermal variation when measured in 3.5-T external field (Fig. 1). It exhibits a bell-shaped anomaly with a pronounced divergence between ZFCW and FCC data. The steep rise in the magnetization by $650 \%$, when temperature is increased by $5 \mathrm{~K}$, indicates a sudden development of a highmagnetization phase stimulated by temperature change in an applied field of $3.5 \mathrm{~T}$, whereas in $0.75 \mathrm{~T} \mathrm{La}_{0.9} \mathrm{Ce}_{0.1} \mathrm{Fe}_{12} \mathrm{~B}_{6}$ compound remains in a low-magnetization state. The spectacular increase of magnetization on the low-temperature side of the 3.5-T ZFCW curve is associated with the onset of ferromagnetic ordering. Throughout this phase transformation, both magnetically ordered phases, AFM and FM, coexist in $\mathrm{La}_{0.9} \mathrm{Ce}_{0.1} \mathrm{Fe}_{12} \mathrm{~B}_{6}$, hence forming a magnetically heterogeneous state or magnetic-phase-segregated state in a chemically homogeneous compound. The bell-like feature correlates with the existence of both low-temperature AFMFM and high-temperature FM-PM magnetic transitions. The intermetallic compound $\mathrm{La}_{0.9} \mathrm{Ce}_{0.1} \mathrm{Fe}_{12} \mathrm{~B}_{6}$ transforms only partially into the FM state when cooled in a 3.5-T external magnetic field. This can be seen in Fig. 1 from the intermediate magnetization plateau value of about $14 \mu_{\mathrm{B}} / \mathrm{f}$.u. During cooling, the magnetization increases as the temperature is lowered and then saturates, demonstrating that the magnetic transformation in $\mathrm{La}_{0.9} \mathrm{Ce}_{0.1} \mathrm{Fe}_{12} \mathrm{~B}_{6}$ depends on the direction of the temperature change. Furthermore, the maximum value of magnetization in the 3.5-T isofield curve is smaller for the FCC branch than for the ZFCW leg. This aspect is rather unusual for standard ferromagnetic materials in an external magnetic field as high as $3.5 \mathrm{~T}$. A similar bell-like behavior was reported for $\mathrm{LaFe}_{12} \mathrm{~B}_{6}$ in the magnetic field interval from 4.75 to $7 \mathrm{~T}$ [17]. Another noteworthy experimental observation in the thermomagnetic data of Fig. 1 is the large thermal hysteresis near the Curie point, which bears witness to the first-order character of the magnetic phase transition.

The isothermal magnetization curves, $M(H)$, of $\mathrm{La}_{0.9} \mathrm{Ce}_{0.1} \mathrm{Fe}_{12} \mathrm{~B}_{6}$ at different fixed temperatures are displayed in Fig. 2. For each isotherm, the applied magnetic field was cycled twice between 0 and the maximum attainable value of $10.5 \mathrm{~T}$ (measurements for the second field reduction are not presented in Fig. 2 for the sake of clarity). Only a selection of the results of the measurements performed at some chosen representative temperatures in the different magnetic states are shown in Fig. 2 (but all of the collected magnetization data were used to construct the magnetic phase diagram depicted in Fig. 14). Each magnetization isotherm starts from the virgin state after zero magnetic field cooling from the paramagnetic region. Figure 2(a) illustrates the data at temperatures of 2 and $10 \mathrm{~K}$, and Fig. 2(b) represents the isotherms taken at temperatures ranging between 20 and $65 \mathrm{~K}$. The first magnetization curves (virgin curves) display a magnetic field-induced metamagnetic transition between the AFM ground state and the FM state below $T_{\mathrm{N}}$ and from the PM into FM states above $T_{\mathrm{N}}$. This observed metamagnetic transition proceeds through a gradual transformation of the PM or AFM phases into FM domains with increasing magnetic field. The magnetization isotherms show a very large magnetic hysteresis, confirming the first-order nature of the metamagnetic phase transition. The width of the hysteresis amounts to $3.5 \mathrm{~T}$ at $10 \mathrm{~K}$ and progressively decreases with increasing temperature.

As can be seen from Fig. 2, during the first application of the magnetic field, at $2 \mathrm{~K}$ the magnetization exhibits two sharp steps (at $\mu_{0} H_{\mathrm{cr} 1}=4.4 \mathrm{~T}$ and $\mu_{0} H_{\mathrm{cr} 2}=7.1 \mathrm{~T}$ ) followed by plateaus, indicating a staircaselike metamagnetic transition similar to that evidenced in the parent compound $\mathrm{LaFe}_{12} \mathrm{~B}_{6}$. This avalanchelike magnetization process arises 

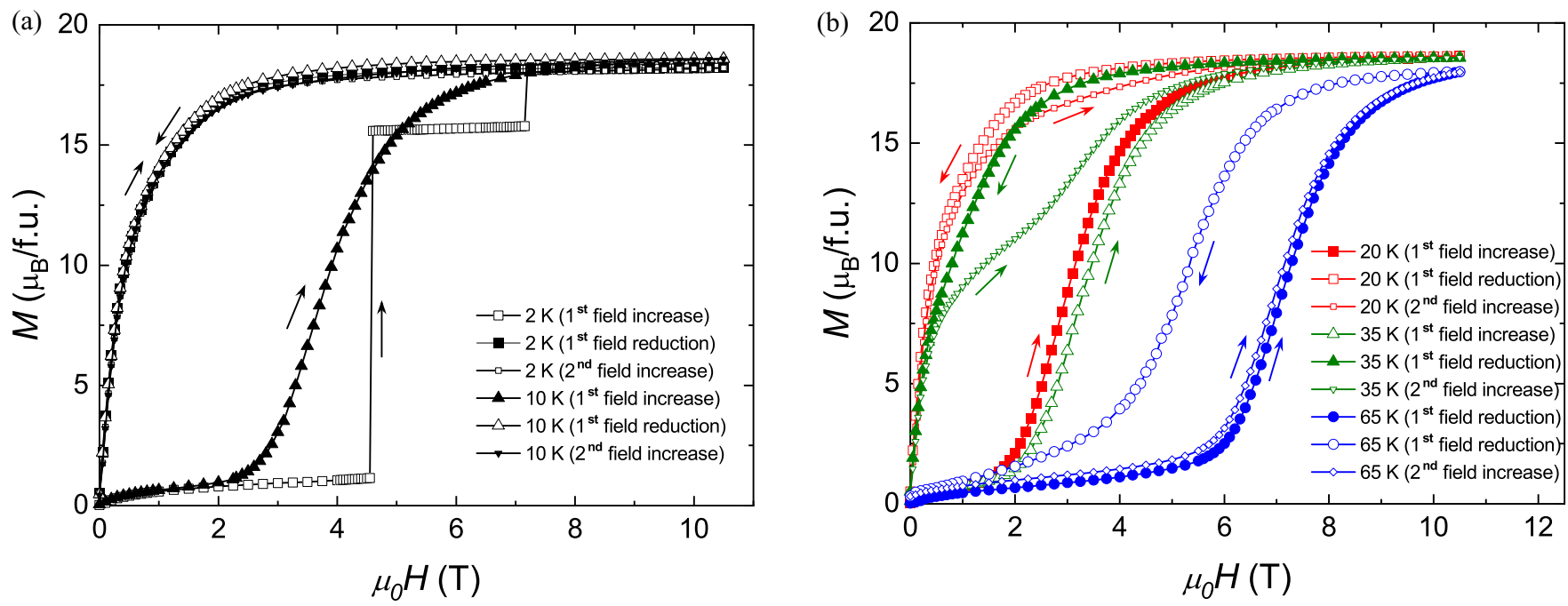

FIG. 2. Isothermal magnetization curves of $\mathrm{La}_{0.9} \mathrm{Ce}_{0.1} \mathrm{Fe}_{12} \mathrm{~B}_{6}$ at (a) 2 and $10 \mathrm{~K}$, and (b) 20, 35, and $65 \mathrm{~K}$.

from transformation of fraction of the specimen volume from the AFM state into the FM state. The first magnetization plateau observed at $2 \mathrm{~K}$ in Fig. 2 represents a magnetically heterogeneous state corresponding to a mixture of the fielddriven FM and original AFM phases (phase separated into AFM and FM domains). During the first step the total magnetic moment of the compound spontaneously increases from 1.15 to $15.59 \mu_{\mathrm{B}} /$ f.u. At the second metamagnetic jump it changes from $15.78 \mu_{\mathrm{B}} /$ f.u. to the saturation moment of the fully FM polarized state, $18.22 \mu_{\mathrm{B}} /$ f.u. In $\mathrm{La}_{0.9} \mathrm{Ce}_{0.1} \mathrm{Fe}_{12} \mathrm{~B}_{6}$, the ultrasharp magnetization jumps are only seen in the virgin magnetization curve and are absent in the subsequent envelope. The multiple abrupt steps are restricted to very low temperatures and disappear at $8 \mathrm{~K}$ where the magnetization process becomes smooth.

During the second magnetic field increase in the temperature range between 2 and $25 \mathrm{~K}$, the curve presents a conventional ferromagneticlike magnetization process and practically coincides with the first demagnetization loop. The first-order AFM-FM transformation is fully irreversible in this temperature region, i.e., after the applied field is removed, almost $100 \%$ of the $\mathrm{La}_{0.9} \mathrm{Ce}_{0.1} \mathrm{Fe}_{12} \mathrm{~B}_{6}$ alloy remains in the induced-FM state. In contrast, when the temperature is increased above $25 \mathrm{~K}$ and below $55 \mathrm{~K}$, the shape of the $M(H)$ curves during the second increase of the external field from 0 to $10.5 \mathrm{~T}$ is even more complex and quite unusual when compared to the behavior observed below $25 \mathrm{~K}$. The magnetization shows a ferromagneticlike dependence (see the low magnetic field portion at $35 \mathrm{~K}$ ) followed by a metamagnetic phase transformation. From $\sim 25$ to $\sim 55 \mathrm{~K}$, the magnetic field-induced AFM-FM and PM-FM transitions are partially reversible; a fraction of the sample volume recovers the initial AFM or PM state when the magnetic field is brought back to zero. Both reversible and irreversible transitions are present in the temperature interval between $\sim 25$ and $\sim 55 \mathrm{~K}$, and the fraction of the system, which undergoes the irreversible transformation, decreases with temperature. Beyond $55 \mathrm{~K}$, the PM-FM metamagnetic transition becomes completely reversible, but accompanied with a magnetic hysteresis.

\section{B. Thermal expansion and magnetostriction}

$\mathrm{La}_{0.9} \mathrm{Ce}_{0.1} \mathrm{Fe}_{12} \mathrm{~B}_{6}$ exhibits successive magnetic transformations and a remarkable metamagnetic transition which is characterized by an enormous magnetization variation and a huge hysteresis. Consequently, $\mathrm{La}_{0.9} \mathrm{Ce}_{0.1} \mathrm{Fe}_{12} \mathrm{~B}_{6}$ is expected to display large magnetovolume effects across the magnetic transitions. To explore more profoundly the nature of this unusual magnetic behavior (first-order character of the AFM-FM and FM-PM transitions), LTE $(\Delta L / L)$ was measured in various applied magnetic fields following the same experimental procedure as in the temperature dependence of the magnetization described above. The obtained results are presented in Fig. 3. An anomaly is found at $\approx 33 \mathrm{~K}$ in the spontaneous $\left(\mu_{0} H=0 \mathrm{~T}\right)$ LTE curve; this value is in good agreement with the Néel temperature deduced from thermomagnetic measurements. No difference was detected between the heating and cooling protocols. In zero magnetic field, the compound expands upon heating (positive thermal expansion) at temperatures exceeding $T_{\mathrm{N}}$; however, below the Neel temperature $\Delta L / L$ increases as the temperature is lowered (negative thermal expansion, NTE). No significant variation in $\Delta L / L$ was observed at $T_{\mathrm{N}}$, suggesting weak magnetoelastic effects associated with the second-order AFM-PM transition.

The LTE curve $\left(\mu_{0} H=3 \mathrm{~T}\right)$ manifests a bell-like behavior and magnetic events which are very much similar to the phase transitions found in the isofield magnetization data. Upon heating in a 3-T magnetic field, the zero-field cooled sample displays a crossover from positive to negative thermal expansion due to the AFM-FM and FM-PM transitions. During cooling $\left(\mu_{0} H=3 \mathrm{~T}\right)$, the lattice undergoes an expansion and then $\Delta L / L$ saturates at low temperatures. Interestingly, a sudden jump is detected in the 4-T LTE curve; $\Delta L / L$ varies abruptly from 0.025 to $0.14 \%$ when the temperature changes by only $0.1 \mathrm{~K}$. This result reveals that at $4 \mathrm{~T}$, the increase 

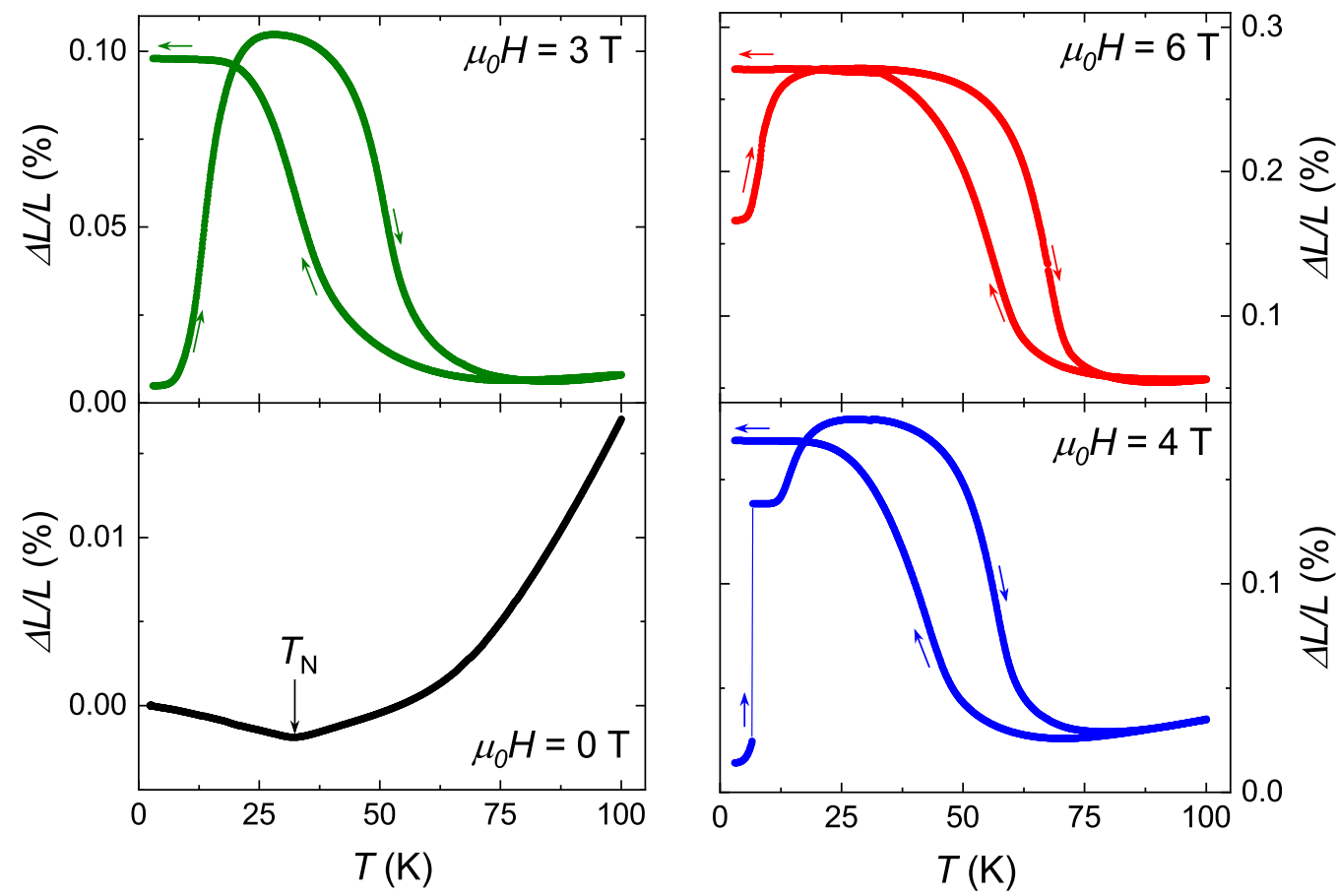

FIG. 3. Linear thermal expansion of $\mathrm{La}_{0.9} \mathrm{Ce}_{0.1} \mathrm{Fe}_{12} \mathrm{~B}_{6}$ as a function of temperature recorded in magnetic fields of $0,3,4$, and $6 \mathrm{~T}$.

in temperature triggers the AFM-FM magnetic phase transformation at low temperatures. Like the magnetization, the forced magnetostriction curves recorded in magnetic fields $\mu_{0} H=3,4$, and $6 \mathrm{~T}$ also show a pronounced splitting and a huge thermal hysteresis of $\sim 15 \mathrm{~K}$ between the ZFCW and FCC modes. The large length variation and temperature hysteresis indicate the existence of important structural effects across the first-order AFM-FM and FM-PM transitions. In the 6-T ZFCW data, a NTE phenomenon is observed over a temperature interval of $\Delta T \sim 60 \mathrm{~K}$ yielding an average linear thermal expansion coefficient $\alpha_{L}=(1 / L)(\Delta L / \Delta T)=$ $-37 \times 10^{-6} \mathrm{~K}^{-1}$. Such a large NTE coefficient compares well with the value obtained for the itinerant-electron metamagnetic compound $\mathrm{LaFe}_{10.5} \mathrm{CoSi}_{1.5} \alpha_{\mathrm{L}}=-26 \times 10^{-6} \mathrm{~K}^{-1}[36]$. It is important to emphasize that the absolute value of $\alpha_{\mathrm{L}}$ for $\mathrm{La}_{0.9} \mathrm{Ce}_{0.1} \mathrm{Fe}_{12} \mathrm{~B}_{6}$ is about 4 times larger than that of $\mathrm{ZrW}_{2} \mathrm{O}_{8}$, $\alpha_{L}=-9 \times 10^{-6} \mathrm{~K}^{-1}$, which is currently used as commercial NTE material [37].

To clarify the volume change by the metamagnetic phase transition, longitudinal $\left(\Delta L / L_{\|}\right)$and transverse $\left(\Delta L / L_{\perp}\right)$ magnetostriction isotherms were recorded. Using these data, the volume magnetostriction $\left(\Delta V / V=\Delta L / L_{\|}+2 \Delta L / L_{\perp}\right)$ and the anisotropic magnetostriction $\left(\Delta L / L_{\|}-\Delta L / L_{\perp}\right)$ were assessed. In Fig. 4(a) we present the forced-volume magnetostriction isotherms at various temperatures in the AFM region. For all isotherms the sample was cooled in zero magnetic field. At $4 \mathrm{~K}$ in the field-increasing branch, a sharp discontinuity of $\Delta V / V \approx 0.65 \%$ occurs at a certain critical field $\mu_{0} H_{\mathrm{cr}}=4.2 \mathrm{~T}$. No anomaly is detected in the reverse leg and $\Delta V / V$ keeps an almost constant value down to the zero magnetic field point, i.e., the material remains in the final deformation state. This demonstrates the fully irreversible nature of the phase transition at $4 \mathrm{~K}$. This strong magnetic field-induced irreversibility is an outstanding fea- ture and consistent with the irreversibility observed in the isothermal magnetization curves. The jump present in the magnetostriction isotherm is similar to those seen in the virgin magnetization loops and proves the strong correlation between magnetic and elastic properties in $\mathrm{La}_{0.9} \mathrm{Ce}_{0.1} \mathrm{Fe}_{12} \mathrm{~B}_{6}$ intermetallic compound. A huge magnetic hysteresis is evidenced, demonstrating that the phase transformation is of first-order type in nature. The magnetostriction results clearly attest that the metamagnetic phase transition is accompanied by large magnetovolume effects. In other words the applied magnetic field induces a transition from a low-volume, lowmagnetization phase to a high-volume, high-magnetization phase. The thermal variation of the volume and anisotropic magnetostriction at the maximum attainable field of $6 \mathrm{~T}$ is depicted in Fig. 4(b). In the present case a large anisotropic effect is observed, i.e., the value of the longitudinal magnetostriction is lower than that of the transverse one $\left(\Delta L / L_{\perp}>\Delta L / L_{\|}\right)$. The anisotropic magnetostriction is as large as $(10 \mathrm{~K}, 6 \mathrm{~T}) \approx$ $-0.10 \%$, but the magnetostriction isotherms are not saturated even under $6 \mathrm{~T}$. The maximum volume magnetostriction associated with the AFM-FM transition is estimated to be about $\Delta V / V(25 \mathrm{~K}, 6 \mathrm{~T}) \approx 1.15 \%$. When this latter value is compared with the volume change calculated from LTE data of Fig. 3, $\Delta V / V=3 \Delta L / L=0.65 \%$, one notices a significant discrepancy between them. This large difference in the observed relative volume variations, when taken into account concurrently with the presence of a huge anisotropic magnetostriction, likely reflects the role of anisotropy in describing the magnetoelastic effects of the compound. It is noteworthy that the relative volume change, $\Delta V / V(25 \mathrm{~K}, 6 \mathrm{~T}) \approx$ $1.15 \%$, in $\mathrm{La}_{0.9} \mathrm{Ce}_{0.1} \mathrm{Fe}_{12} \mathrm{~B}_{6}$ is comparable in magnitude to the isotropic forced-volume magnetostriction due to the metamagnetic transition in iron-rich itinerant-electron compounds such as $\mathrm{La}\left(\mathrm{Fe}_{x} \mathrm{Al}_{1-x}\right)_{13}$ [38] and $\mathrm{La}\left(\mathrm{Fe}_{x} \mathrm{Si}_{1-x}\right)_{13}$ [39]. 

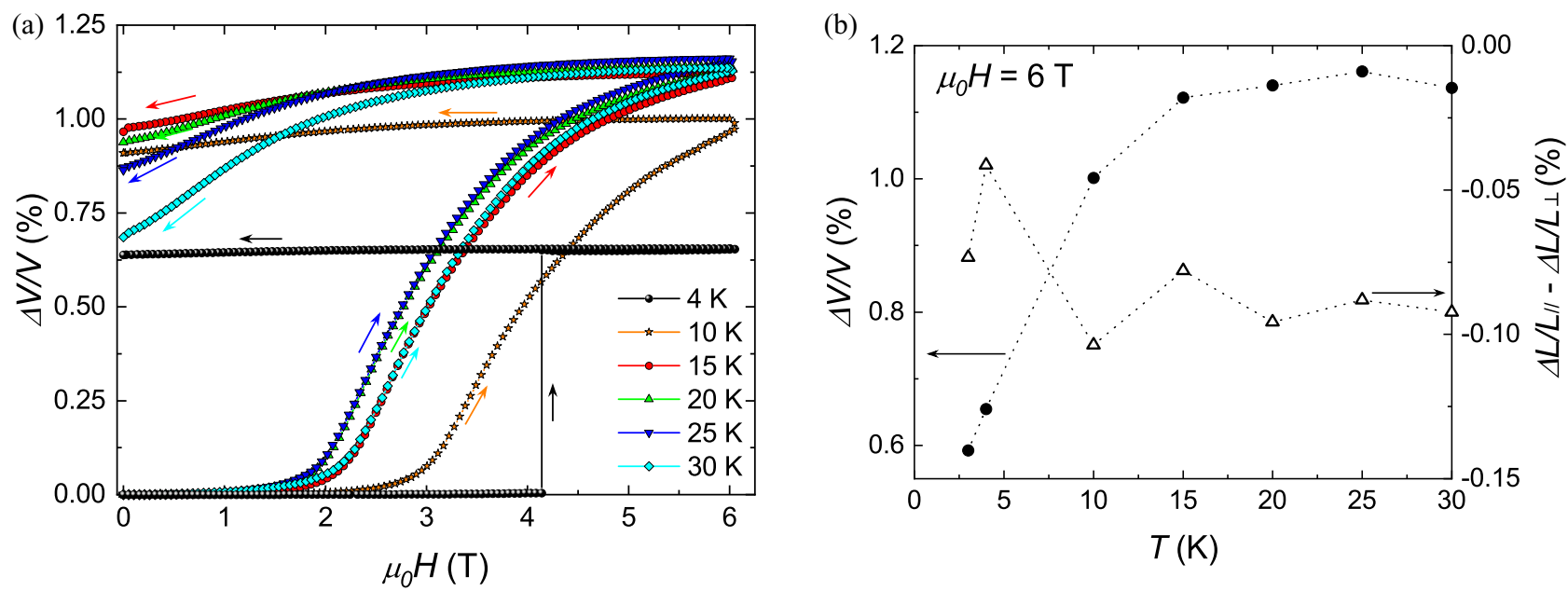

FIG. 4. (a) Volume magnetostriction isotherms measured between 4 and $30 \mathrm{~K}$. (b) Temperature dependence of the volume $(\Delta V / V=$ $\left.\Delta L / L_{\|}+2 \Delta L / L_{\perp}\right)$ and anisotropic $\left(\Delta L / L_{\|}-\Delta L / L_{\perp}\right)$ magnetostriction measured for $\mathrm{La}_{0.9} \mathrm{Ce}_{0.1} \mathrm{Fe}_{12} \mathrm{~B}_{6}$ at the maximum applied field of $6 \mathrm{~T}$.

Indeed, Irisawa et al. [38] reported a value of $\Delta V / V=1 \%$ for $\mathrm{La}\left(\mathrm{Fe}_{0.87} \mathrm{Al}_{0.13}\right)_{13}$. Volume changes of 0.9 and $1.5 \%$ were obtained for $\mathrm{La}\left(\mathrm{Fe}_{0.86} \mathrm{Si}_{0.14}\right)_{13}$ and $\mathrm{La}\left(\mathrm{Fe}_{0.88} \mathrm{Si}_{0.12}\right)_{13}$, respectively [39].

\section{Temperature and magnetic field-dependent $x$-ray diffraction}

The consistence found in the magnetostriction and magnetization data obviously reflects the strong coupling between lattice and spin degrees of freedom in $\mathrm{La}_{0.9} \mathrm{Ce}_{0.1} \mathrm{Fe}_{12} \mathrm{~B}_{6}$. The magnetostriction data suggest strong structural effects associated with the magnetic transitions when the applied field exceeds the critical value. Therefore, the crystal structure of the field-induced FM phase cannot be inferred from zero magnetic field diffraction spectra since the temperature alone cannot trigger the transformation. Magnetic field-dependent $\mathrm{x}$-ray powder diffraction appears to be the viable option to provide structural details at the atomic scale and to decipher how a crystal lattice is responsible for a specified magnetic behavior. In order to get a deeper insight into the nature of the low-temperature transformation in this intriguing material $\mathrm{La}_{0.9} \mathrm{Ce}_{0.1} \mathrm{Fe}_{12} \mathrm{~B}_{6}$, x-ray diffraction measurements under applied magnetic fields were performed down to $15 \mathrm{~K}$.

The diffraction patterns recorded in zero magnetic field $\left(\mu_{0} H=0 \mathrm{~T}\right)$ were identical in the temperature range from 300 to $15 \mathrm{~K}$ apart from an anisotropic shift of Bragg reflections due to thermal expansion. According to the Rietveld analyses the compound had the rhombohedral $\mathrm{SrNi}_{12} \mathrm{~B}_{6}$-type structure (space group $R \overline{3} \mathrm{~m}$ ) over the entire investigated temperature range. The crystal symmetry is preserved down to $15 \mathrm{~K}$, i.e., the trigonal symmetry of the atomic arrangement is kept unchanged. No indication of a temperature-induced structural transition was detected down to the lowest temperature of the x-ray diffraction experiment. The lattice parameters $a$ and $c$, unit-cell volume $V$, and $c / a$ ratio of the rhombohedral cell are plotted against temperature in Fig. 5. The zero-field thermodiffraction results reveal that both lattice constants are anisotropically reduced upon cooling, thus leading to a diminution in the cell volume. On the other hand, the $c / a$ ratio increases as the temperature is lowered. At temperatures ranging from 300 to $125 \mathrm{~K}$, the lattice constants vary linearly and the calculated coefficients of linear thermal expansion along the two principal crystallographic directions are $\alpha_{a}=$ $14.30 \times 10^{-6} \mathrm{~K}^{-1}$ and $\alpha_{c}=7.29 \times 10^{-6} \mathrm{~K}^{-1}$. Along the $a$-axis direction (in the basal plane) the thermal expansion is much larger than that along the threefold symmetry axis $c$. The crystallographic volume thermal expansion coefficient amounts to $\alpha_{V}=36 \times 10^{-6} \mathrm{~K}^{-1}$. Below $50 \mathrm{~K}$, the unit-cell dimension $a$ is basically constant and therefore the LTE along this particular crystallographic axis approaches zero, whereas the LTE along the high-symmetry direction $c$ becomes almost negligible only below $25 \mathrm{~K}$. Even though the practically zero thermal expansion found along the two principal crystallographic directions at low temperatures is in agreement with the normal phonon contribution becoming insignificant (consistent with Grüneisen's law), the difference in the temperature at which the LTE approaches zero is in accordance with the anisotropy of the crystal lattice. No perceptible anomaly of thermal expansion is observed around $T_{\mathrm{N}}$ in the temperature dependence of the unit-cell volume.

In order to examine the lattice response to the magnetic transitions, x-ray diffraction diagrams were recorded at temperatures ranging between 15 and $100 \mathrm{~K}$ in a constant applied magnetic field. Diffractograms collected at $15 \mathrm{~K}$ in magnetic fields of 0 and $5.5 \mathrm{~T}$ are plotted in Fig. 6(a). For clarity reasons, only the angular $2 \theta$ range $14^{\circ}-30^{\circ}$ is displayed. From the visual inspection of the x-ray powder diffraction patterns, it is readily apparent that there are big differences between them. The diffraction profile is considerably altered as the applied magnetic field increases (above $1.75 \mathrm{~T}$ ) and new Bragg reflections appear, indicating the occurrence of a structural phase transition. At $5.5 \mathrm{~T}$, these new diffraction peaks become dominant and a coexistence of two crystallographically different $\mathrm{La}_{0.9} \mathrm{Ce}_{0.1} \mathrm{Fe}_{12} \mathrm{~B}_{6}$ phases is observed, consistent with the first-order character of the phase transformation. The complete, fully refined $\mathrm{x}$-ray spectra recorded at $15 \mathrm{~K}$ in 0 and 5.5-T applied field are shown in Figs. 6(b) and 6(c), respectively. As noted above, only the rhombohedral phase (henceforth called the Rhom phase) with $R \overline{3} m$ space 

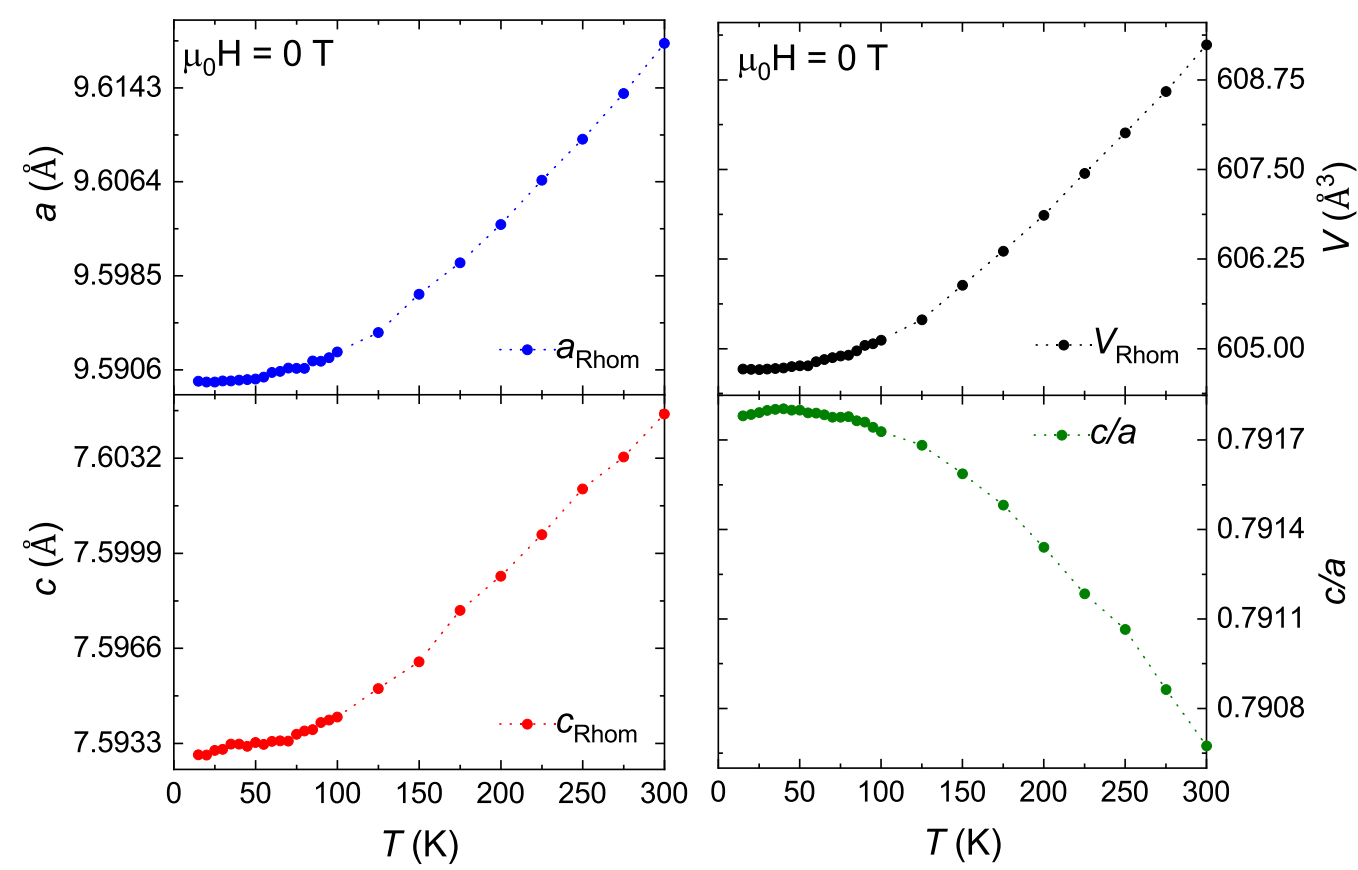

FIG. 5. Temperature dependence of the lattice parameters $a$ and $c$, unit-cell volume $V$, and $c / a$ ratio of the rhombohedral cell determined from Rietveld refinement of x-ray powder diffraction patterns measured during cooling in $\mu_{0} H=0 \mathrm{~T}$.

group is present at $0 \mathrm{~T}$ in the AFM state at $15 \mathrm{~K}$. The new Bragg lines seen in the diffraction pattern collected at $5.5 \mathrm{~T}$ can be indexed in a monoclinic cell adopting the $C 2 / \mathrm{m}$ space group (henceforth called the Mono phase). A good refinement of the structure was achieved in $C 2 / \mathrm{m}$ symmetry group. The Rietveld-refined structural parameters are summarized in Table I. In the International Tables for Crystallography, all of the listed maximal nonisomorphic subgroups for $R \overline{3} m$ are trigonal except for one, which is the monoclinic space-group symmetry $C 2 / m$. This crystal symmetry, $C 2 / m$, is used to describe the lattice distortion and it fits very well the diffraction pattern shown in Fig. 6(c). Therefore, it is likely to be the correct high magnetic field crystallographic structure of the $\mathrm{La}_{0.9} \mathrm{Ce}_{0.1} \mathrm{Fe}_{12} \mathrm{~B}_{6}$ compound. The threefold symmetry in the $a b$ basal plane is broken in the magnetic field-induced state and such structural distortion lowers the lattice symmetry from trigonal to monoclinic.

TABLE I. Structural parameters of $\mathrm{La}_{0.9} \mathrm{Ce}_{0.1} \mathrm{Fe}_{12} \mathrm{~B}_{6}$ obtained from Rietveld refinement of the x-ray powder diffraction patterns collected at $T=15 \mathrm{~K}$ in magnetic field of 0 and $5.5 \mathrm{~T}$. Crystallographic space group, lattice parameters, and unit-cell volume are indicated.

\begin{tabular}{lccc}
\hline \hline & $15 \mathrm{~K}$ & $15 \mathrm{~K}$ & $15 \mathrm{~K}$ \\
& $0 \mathrm{~T}$ & $5.5 \mathrm{~T}$ & $5.5 \mathrm{~T}$ \\
\hline Space group & $R-3 m$ & $R-3 m$ & $C / 2 m$ \\
$a(\AA)$ & $9.5901(4)$ & $9.5827(5)$ & $7.5758(6)$ \\
$b(\AA)$ & $9.5901(4)$ & $9.5827(5)$ & $9.7824(6)$ \\
$c(\AA)$ & $7.5924(5)$ & $7.5979(6)$ & $5.9664(4)$ \\
$\beta\left(\left(^{\circ}\right)\right.$ & 90 & 90 & $113.085(11)$ \\
$V\left(\AA^{3}\right)$ & $604.72(5)$ & $604.23(44)$ & $406.76(7)$ \\
\hline \hline
\end{tabular}

Let us describe briefly the relationship between the rhombohedral (in hexagonal setting) and monoclinic lattices. The space-group symmetry $C 2 / m$ is one of the maximal nonisomorphic subgroups of the parent $R \overline{3} m$ crystal symmetry group and the relation between the monoclinic unit cell and the rhombohedral (hexagonal) unit cell is as follows:

$$
(\boldsymbol{a b c})_{\text {Mono }}=(\boldsymbol{a b c})_{\mathrm{Rhom}}\left(\begin{array}{ccc}
-1 / 3 & 1 & 1 / 3 \\
1 / 3 & 1 & -1 / 3 \\
-2 / 3 & 0 & -1 / 3
\end{array}\right) .
$$

The rhombohedral and monoclinic unit-cell volumes are related in accordance with: $V_{\text {Mono }}(C / 2 m)=2 / 3 V_{\text {Rhom }}(R \overline{3} m)$. The observed crystal structure change to $C 2 / m$ is induced by magnetic field. Since $C 2 / m$ is a subgroup of $R \overline{3} m$ the first-order character of the transition is not obligatory and it originates from the magnetic transition from AFM ground state to field-induced FM state.

Considering that a different number of atoms are involved in the two crystallographically distinct structures and in order to compare directly their volumes, it is therefore necessary to normalize the cell volumes to a formula unit. The temperature dependence of the volume per chemical formula in an applied magnetic field of $5.5 \mathrm{~T}$ is illustrated in Fig. 7 including the values of the two crystal structures. In association with the field-induced metamagnetic phase transition, the analysis of the x-ray diffraction diagrams demonstrates that in $5.5 \mathrm{~T}$ $\mathrm{La}_{0.9} \mathrm{Ce}_{0.1} \mathrm{Fe}_{12} \mathrm{~B}_{6}$ exhibits an incomplete crystallographic transformation from a rhombohedral (AFM, PM) to a monoclinic (FM) structure. The powder diffraction results reveal the existence of a wide temperature interval of coexistence of the monoclinic and rhombohedral lattices. At $15 \mathrm{~K}$, an applied magnetic field of $5.5 \mathrm{~T}$ is capable to induce $\sim 90 \%$ of the structural alteration, and the field-driven Rhom to Mono 

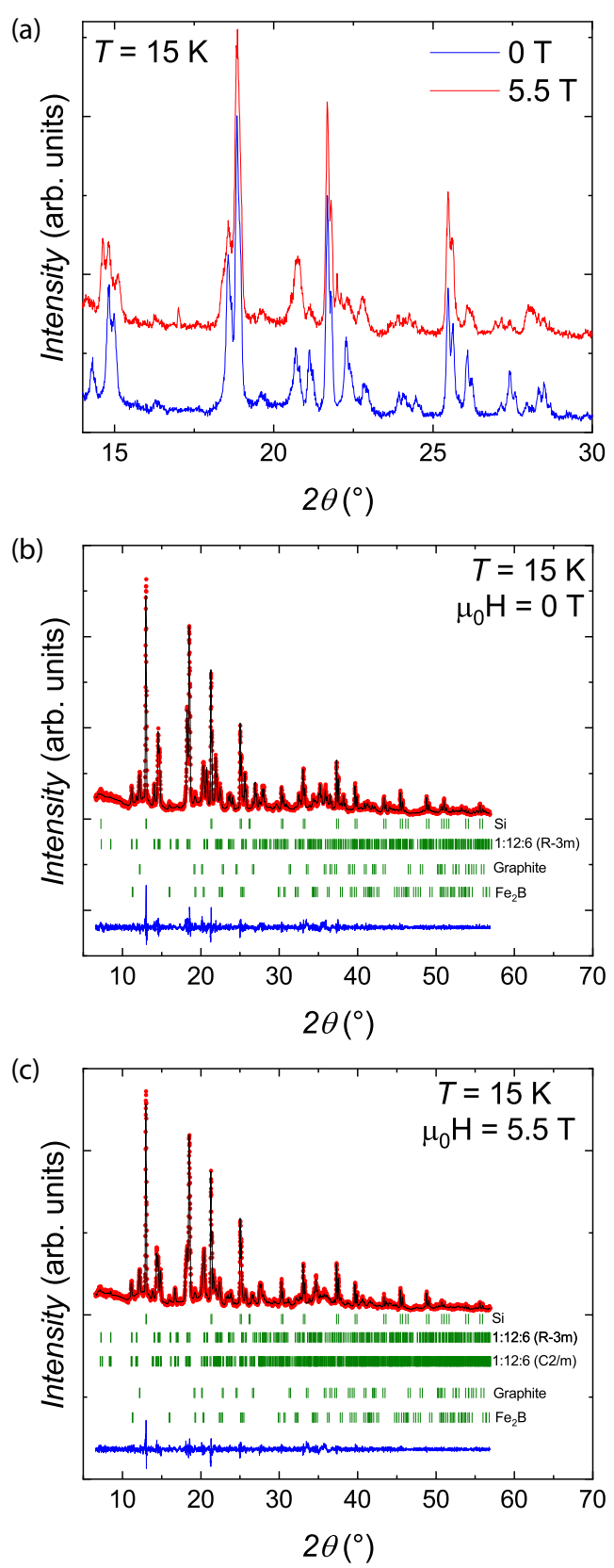

FIG. 6. (a) X-ray powder diffraction patterns collected at $15 \mathrm{~K}$ $\left(\lambda_{\mathrm{K} \alpha 1}=0.70932 \AA\right.$ and $\left.\lambda_{\mathrm{K} \alpha 2}=0.71340 \AA\right)$ in magnetic fields of 0 and $5.5 \mathrm{~T}$. Only the diffraction angle range from $14^{\circ}$ to $30^{\circ}$ is shown to better illustrate development of phases with magnetic field. (b), (c) Rietveld refinements of the $\mathrm{x}$-ray diffraction pattern collected at $15 \mathrm{~K}$ in magnetic field of $\mu_{0} H=0 \mathrm{~T}$ and $\mu_{0} H=5.5 \mathrm{~T}$, respectively. The observed (red dots), calculated (black lines) patterns are shown. The blue line represents the difference $I_{\mathrm{obs}}-I_{\text {calc }}$. The vertical bars (olive) indicate positions of Bragg peaks for the different phases.

crystallographic phase transition is accompanied by unit-cell volume change of $0.85 \%$. At $70 \mathrm{~K}$, the volume of the PM (Rhom) phase is about $0.86 \%$ smaller than that of the forced FM (Mono) phase. The volume changes at the first-order AFM-FM and FM-PM magnetic transitions are nearly the same. A symmetry-lowering crystallographic distortion takes place at the magnetic transitions.

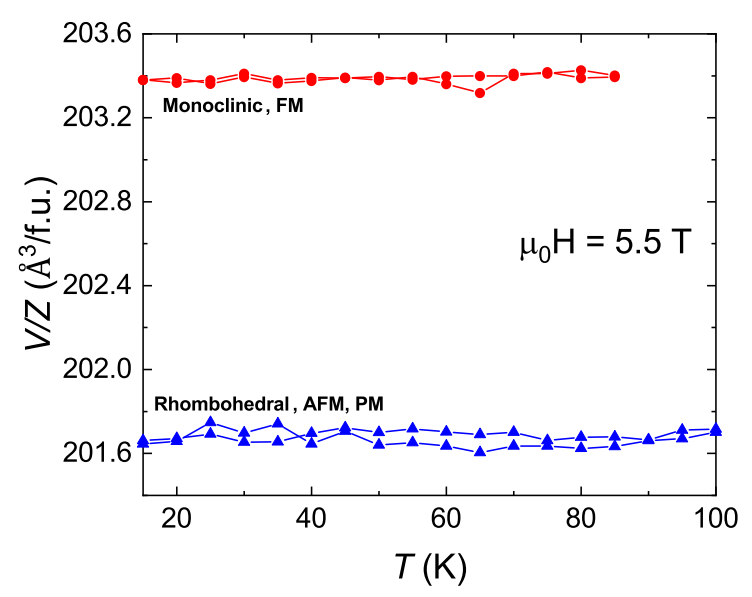

FIG. 7. Temperature dependence of the cell volume per formula unit for $\mathrm{La}_{0.9} \mathrm{Ce}_{0.1} \mathrm{Fe}_{12} \mathrm{~B}_{6}$ (both rhombohedral and monoclinic phases) determined from $\mathrm{x}$-ray powder diffraction measurements during heating and cooling in $\mu_{0} H=5.5 \mathrm{~T}$ applied magnetic field.

The lattices parameters, $a$ and $c$, of the rhombohedral structure are plotted as a function of temperature in Fig. 8. From these data, it is easy to see that the unit-cell expansion in $\mathrm{La}_{0.9} \mathrm{Ce}_{0.1} \mathrm{Fe}_{12} \mathrm{~B}_{6}$ proves highly anisotropic: the lattice expands in the basal plane while it shrinks along the threefold symmetry axis $c$. The results of Fig. 8 reveal another interesting phenomenon, which is a large difference between the thermal hysteresis region in the $a b$ basal plane and that along the $c$ axis. The lattice constant $a$ is hysteretic in the temperature range between $\sim 30$ and $\sim 60 \mathrm{~K}$, while hysteresis along the high-symmetry direction $c$ persists up to $\sim 75 \mathrm{~K}$. This direction dependence of the thermal hysteresis region is consistent with the anisotropy of the rhombohedral lattice. Figure 9 shows the thermal variation of the structural parameters of the monoclinic cell. It is remarkable that the lattice constants $a, b$, and $c$, and the Bragg angle $\beta$ first evolve slowly at low temperatures and then exhibit very sharp changes at the transition point. The variation in cell dimensions presents a

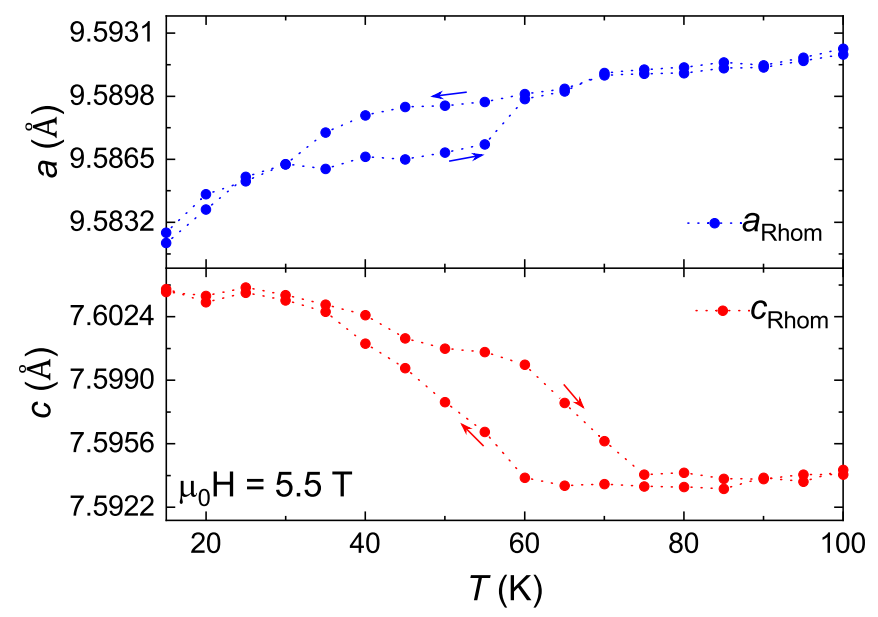

FIG. 8. Temperature dependence of the lattice parameters $a$ and $c$ of the rhombohedral cell determined from $\mathrm{x}$-ray powder diffraction measurements during heating and cooling in $\mu_{0} H=5.5 \mathrm{~T}$ applied magnetic field. 

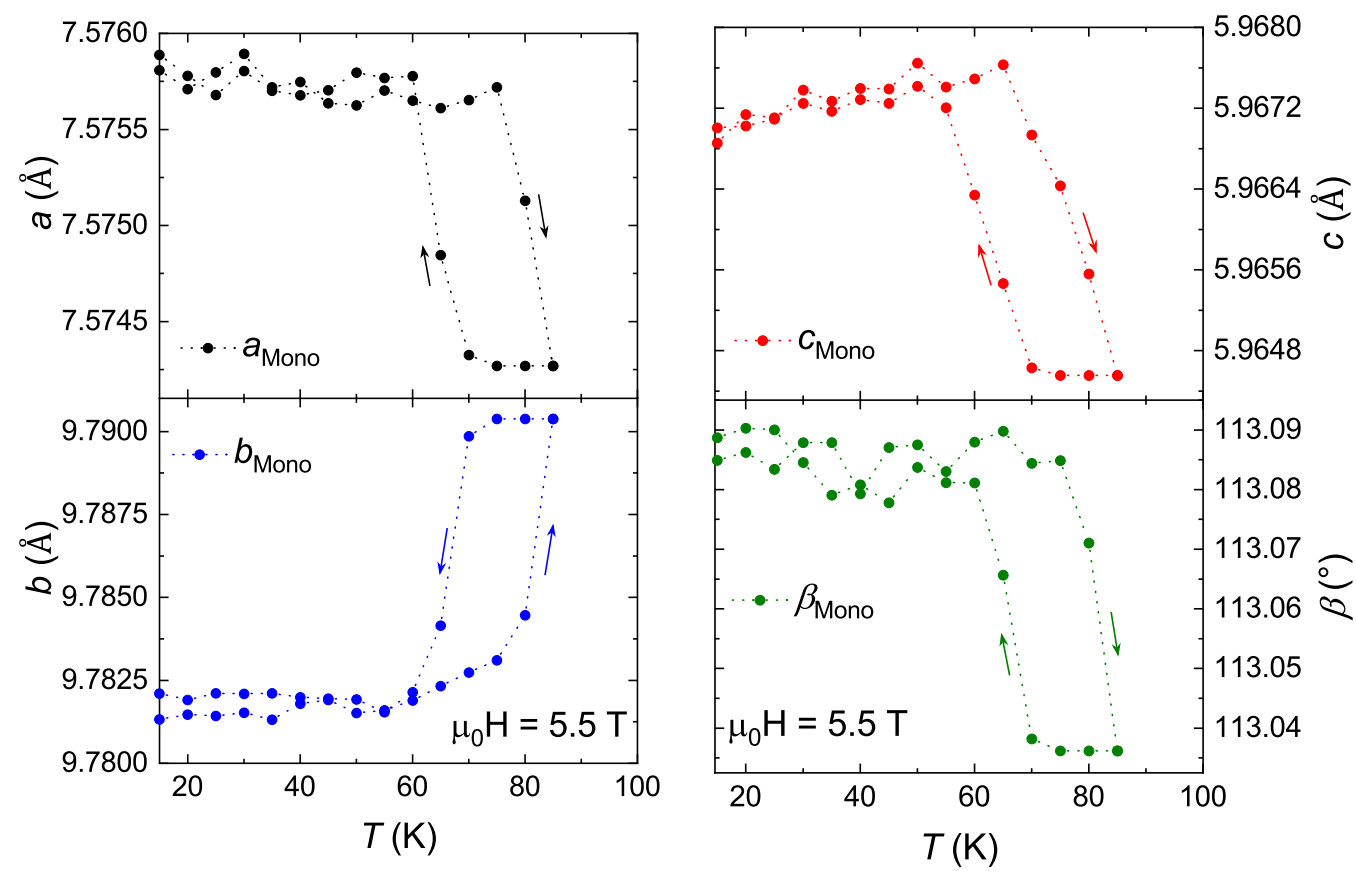

FIG. 9. Temperature dependence of the unit-cell dimensions $a, b$, and $c$, and Bragg angle $\beta$ of the monoclinic phase determined from x-ray powder diffraction measurements during heating and cooling in $\mu_{0} H=5.5 \mathrm{~T}$ applied magnetic field.

strong anisotropy: in the vicinity of the magnetic transition both lattice parameters, $a$ and $c$, decrease while the cell parameter, $b$, increases upon heating. In addition, the structural parameters of both $\mathrm{La}_{0.9} \mathrm{Ce}_{0.1} \mathrm{Fe}_{12} \mathrm{~B}_{6}$ polymorphs display a large thermal hysteresis.

The variation of the concentration of the rhombohedral and monoclinic phases with temperature during both heating and cooling in magnetic fields of $\mu_{0} H=2.5$ and $5.5 \mathrm{~T}$ is reported in Fig. 10. A temperature hysteresis of $20 \mathrm{~K}$ is noticeable between the ZFCW and FCC data collected in $2.5 \mathrm{~T}$. Upon heating, the fraction of induced monoclinic phase in 2.5-T applied field diminishes, amounting to less than $6 \%$ at 70 $\mathrm{K}$. On decreasing the temperature, the transition in $2.5 \mathrm{~T}$ is incomplete with approximately $54 \%$ of the rhombohedral $\mathrm{La}_{0.9} \mathrm{Ce}_{0.1} \mathrm{Fe}_{12} \mathrm{~B}_{6}$ converted into the monoclinic allotrope at $15 \mathrm{~K}$. The system is partially transformed into the monoclinic structure, and the latter becomes the majority component $(\sim 90 \%)$ in the compound at $15 \mathrm{~K}$ and $5.5 \mathrm{~T}$. The $\mathrm{x}$-ray diffraction results clearly indicate that $\mathrm{La}_{0.9} \mathrm{Ce}_{0.1} \mathrm{Fe}_{12} \mathrm{~B}_{6}$ presents crystallographic inhomogeneity (structurally heterogeneous state or coexistence of polymorphs) below $T_{\mathrm{C}}$ on heating and cooling in magnetic fields of 2.5 and 5.5 T. Beyond the Curie point the sample recovers homogeneity and sets fully in the rhombohedral crystallographic structure which is found at room temperature. The ratio between the low- and high- magnetic field crystallographic structures correlates very well with the percentage of the AFM, PM, and FM phases obtained from magnetization in the same applied fields. The data presented in Fig. 10 result from the competition between the growth of the Rhom (AFM, PM) phase with increasing temperature and the diminution of the Mono (FM) component. The ratio and the stability of the two different $\mathrm{La}_{0.9} \mathrm{Ce}_{0.1} \mathrm{Fe}_{12} \mathrm{~B}_{6}$ polymorphs depends both on the strength of the applied magnetic field and the value of temperature, and the direction of the temperature variation. The magnetically and structurally heterogeneous states observed in $\mathrm{La}_{0.9} \mathrm{Ce}_{0.1} \mathrm{Fe}_{12} \mathrm{~B}_{6}$ are very similar to those found in some intermetallic alloys such as $\mathrm{Gd}_{5}\left(\mathrm{Si}_{x} \mathrm{Ge}_{1-x}\right)_{4}[9,10,40-42]$, Si-doped $\mathrm{CeFe}_{2}$ [12], and MnAs [1,43] and also usually seen in the colossal

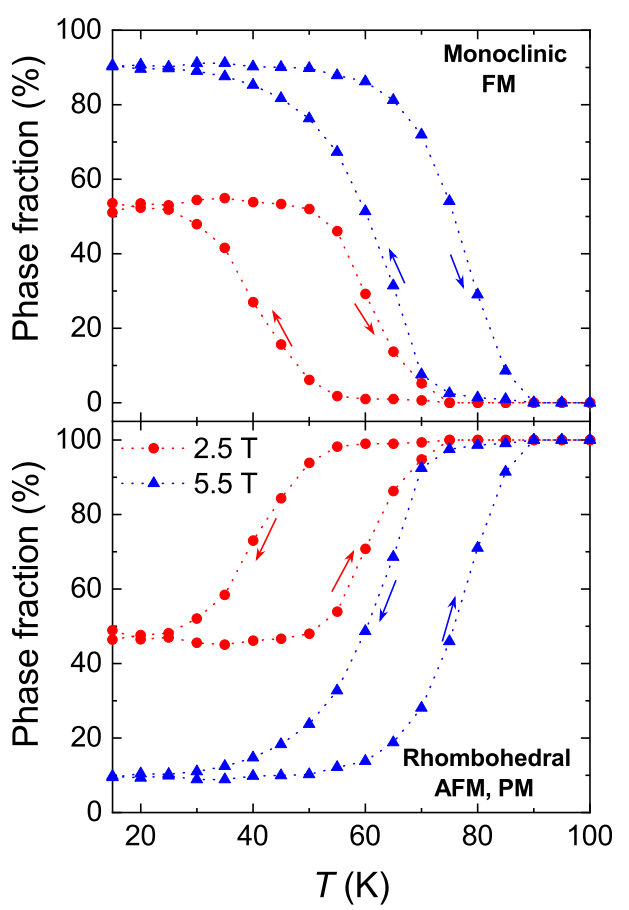

FIG. 10. Temperature dependence of the phase fractions of both rhombohedral and monoclinic crystal structures determined from x-ray powder diffraction measurements during heating and cooling in $\mu_{0} H=2.5$ and $5.5 \mathrm{~T}$ applied magnetic fields. 


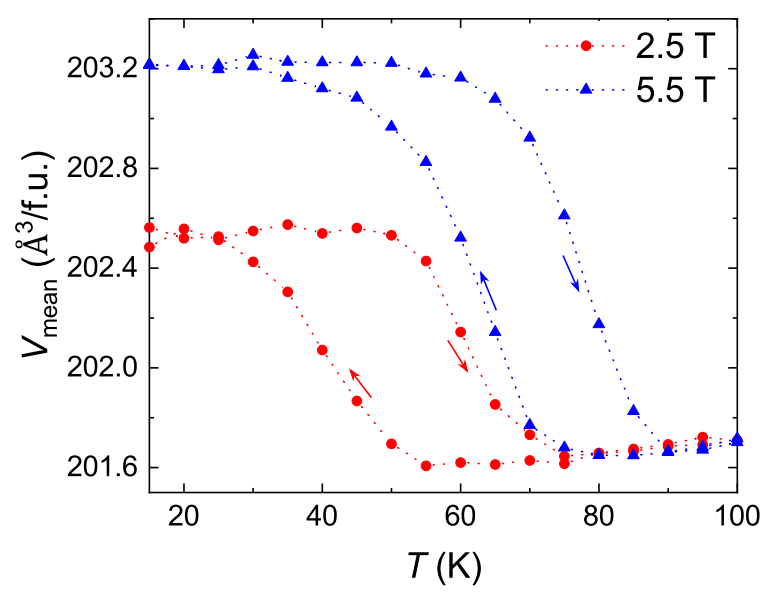

FIG. 11. Temperature dependence of the mean cell volume per formula unit determined from x-ray powder diffraction measurements during heating and cooling in $\mu_{0} H=2.5$ and $5.5 \mathrm{~T}$ applied magnetic fields.

magnetoresistive manganese-based perovskites, where they are often called "phase-segregated states" [44,45].

The thermal dependence of the weighted mean cell volume in 2.5- and 5.5-T applied magnetic fields is illustrated in Fig. 11. The weighted average volume undergoes a transition which is accompanied by a huge thermal hysteresis indicating first-order nature of the phase transition. There is a temperature interval in which the average volume shrinks with increasing temperature; namely, NTE effect occurs over this temperature range. Although the difference in volume between the two polymorphs (monoclinic and rhombohedral phases) is as large as $0.85 \%$, the contraction of the mean volume between 50 and $90 \mathrm{~K}$ is about $0.77 \%$ in 5.5-T magnetic field. The latter value compares well with the relative volume change obtained macroscopically from dilatometric LTE data $\Delta V / V=3 \Delta L / L=0.65 \%$, especially considering anisotropic effects. $\mathrm{La}_{0.9} \mathrm{Ce}_{0.1} \mathrm{Fe}_{12} \mathrm{~B}_{6}$ exhibits a prominent NTE phenomenon over a temperature window of $\Delta T=40 \mathrm{~K}$ with an average volumetric thermal expansion coefficient $\alpha_{V}=-193 \times 10^{-6} \mathrm{~K}^{-1}$. This large NTE effect is generated by the strong spin-lattice coupling. For comparison purposes it is worth noting that the volume thermal expansion coefficient reported for ( $\mathrm{Hf}, \mathrm{Nb}) \mathrm{Fe}_{2}[13]$ and $(\mathrm{Hf}, \mathrm{Ti}) \mathrm{Fe}_{2}$ [46] is at least an order of magnitude smaller than the value obtained here for $\mathrm{La}_{0.9} \mathrm{Ce}_{0.1} \mathrm{Fe}_{12} \mathrm{~B}_{6}$. Our volumetric NTE coefficient compares well with the giant negative thermal expansion observed in ( $\mathrm{Hf}, \mathrm{Ta}) \mathrm{Fe}_{2}$ itinerant-electron metamagnets $\left(\alpha_{V}=-164 \times\right.$ $\left.10^{-6} \mathrm{~K}\right)[47,48]$ and is on the same order of magnitude as the colossal NTE discovered in $\mathrm{Mn}_{0.98} \mathrm{CoGe}\left(\alpha_{V}=-423 \times\right.$ $\left.10^{-6} \mathrm{~K}^{-1}\right)$ [49] and $\mathrm{Bi}_{0.95} \mathrm{La}_{0.05} \mathrm{NiO}_{3}\left(\alpha_{V}=-413 \times 10^{-6} \mathrm{~K}^{-1}\right)$ [50].

To further elucidate the field-induced metamagnetic transition observed in the magnetization and magnetostriction isotherms and in order to get a better understanding of the correlations between the structural and magnetic properties of $\mathrm{La}_{0.9} \mathrm{Ce}_{0.1} \mathrm{Fe}_{12} \mathrm{~B}_{6}$, the magnetic field dependence of the crystallographic structure was also investigated at $15 \mathrm{~K}$. For this study, the sample was cooled in zero magnetic field down to $15 \mathrm{~K}$. After stabilization of the temperature, the external

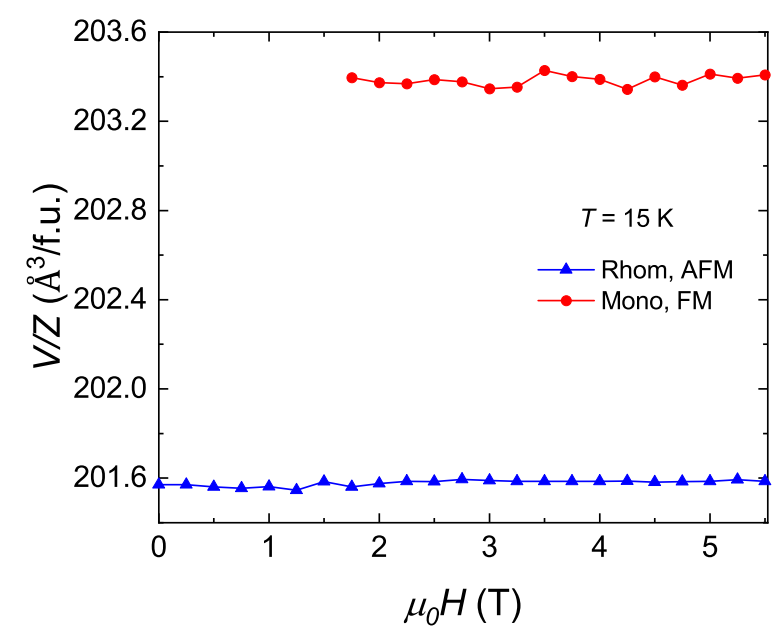

FIG. 12. Magnetic field dependence of the cell volume per chemical formula for $\mathrm{La}_{0.9} \mathrm{Ce}_{0.1} \mathrm{Fe}_{12} \mathrm{~B}_{6}$ at $15 \mathrm{~K}$.

field was then raised at regular steps up to $5.5 \mathrm{~T}$ and $\mathrm{x}$ ray powder diffraction patterns collected at each field step. At $15 \mathrm{~K}$ and $\mu_{0} H<1.75 \mathrm{~T}$, the diffractograms of the zerofield cooled $\mathrm{La}_{0.9} \mathrm{Ce}_{0.1} \mathrm{Fe}_{12} \mathrm{~B}_{6}$ reflect the pure rhombohedral crystal structure. Even though, at all magnetic fields lower than $1.75 \mathrm{~T}$, only the rhombohedral polymorph is detected, the Bragg peaks belonging to the monoclinic phase start to appear at higher applied fields, with dramatic changes of the diffractograms. When the Bragg reflections of the monoclinic structure emerge, the intensities of the diffraction lines that correspond to the rhombohedral phase are reduced.

The field dependences of the volume per chemical formula at $15 \mathrm{~K}$ are displayed in Fig. 12. The Rietveld refinement results reveal that the structure is described solely by the $R \overline{3} m$ crystal symmetry group at low fields while at higher magnetic fields $\left(\mu_{0} H \geqslant 1.75 \mathrm{~T}\right)$ the $\mathrm{x}$-ray diffraction profiles can be well fitted only by including a monoclinic structure. The $C 2 / m$ space-group symmetry is added to model the high-field $\mathrm{x}$-ray powder diffraction data. The volumes for both Rhom (AFM) and Mono (FM) phases are nearly field independent as illustrated in Fig. 12. The volume per formula unit of the AFM phase is smaller than that of FM phase. Our x-ray diffraction results reveal a change of the crystal symmetry at the AFM-FM first-order transition, i.e., a coupled crystallographic-magnetic transformation from a Rhom (AFM) to a Mono (FM) state. In $\mathrm{La}_{0.9} \mathrm{Ce}_{0.1} \mathrm{Fe}_{12} \mathrm{~B}_{6}$ intermetallic compound the crystal and magnetic sublattices are intimately coupled. Therefore, a modification of the magnetic order results in a concomitant change in the crystal lattice. The formation of the FM phase is accompanied by a symmetrylowering lattice distortion.

As a means to follow more precisely the phase transformation, we show in Fig. 13 the weighted mean volume (top panel) and its relative change (bottom panel) at $15 \mathrm{~K}$ as a function of magnetic field. Below 1.75 T, the weighted average volume is almost constant and then raises rapidly as the compound undergoes a magnetic transition just above $1.75 \mathrm{~T}$. The magnetic field-induced metamagnetic transition is also distinctly visible in the relative variation of the volume. The relative change in the mean volume is negligible (approaches 


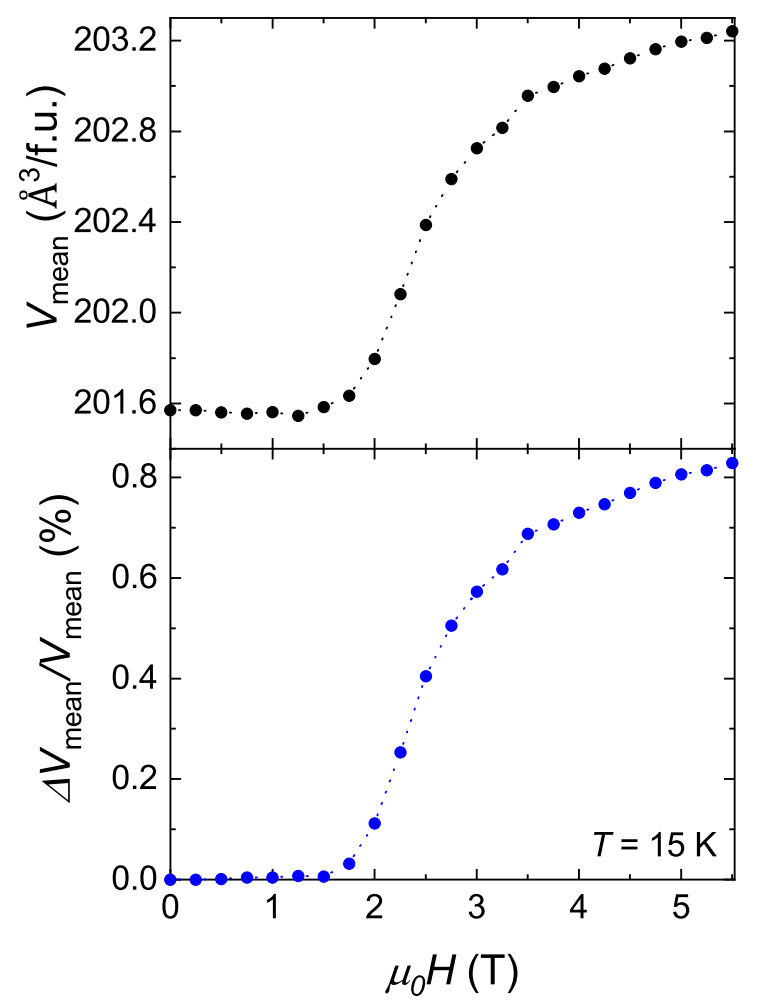

FIG. 13. Magnetic field dependence of the mean cell volume per chemical formula (top) and relative volume change (bottom) determined from $\mathrm{x}$-ray powder diffraction measurements at $15 \mathrm{~K}$.

zero) at low fields, i.e., very weak magnetoelastic effects are detected in the AFM ground state below 1.75 T. The forcedvolume magnetostriction reaches a value of $0.83 \%$ at $5.5 \mathrm{~T}$. This value is reasonably consistent with the relative volume change deduced from the $15 \mathrm{~K}$ dilatometric magnetostriction isotherm $\Delta V / V(5.5 \mathrm{~T}) \approx 1.05 \%$, particularly taking into account the anisotropic behavior. These experimental results reveal that the observed field-induced first-order AFM-FM transformation is concomitant with the symmetry-lowering rhombohedral to monoclinic structural phase transition.

At the local scale the observed martensiticlike transformation should involve different variants as it is expected upon lowering of the crystal symmetry. Due to the crystalline orientation of the shearing-induced variants, several magnetic domains are expected. Further studies enabling to identify the different magnetic domains and crystal variant would be interesting.

\section{Discussion}

The compilation of the experimental results of the magnetization measurements and x-ray diffraction studies is presented in the magnetic-field $\left(\mu_{0} H\right)$-temperature $(T)$ phase diagram of $\mathrm{La}_{0.9} \mathrm{Ce}_{0.1} \mathrm{Fe}_{12} \mathrm{~B}_{6}$ intermetallic compound (Fig. 14). The critical transition field of the downward-field scan of the metamagnetic transition, $\mu_{0} H_{\mathrm{cr} \text {,down }}$, is approximately zero at 35 $\mathrm{K}$ and increases monotonically upon heating. However, the temperature dependence of the transition field obtained for the ascending-field process, $\mu_{0} H_{\mathrm{cr} \text {, up }}$, is nonmonotonic. Below 25 $\mathrm{K} \mu_{0} H_{\mathrm{cr} \text {,up }}$ diminishes with increasing temperature while it

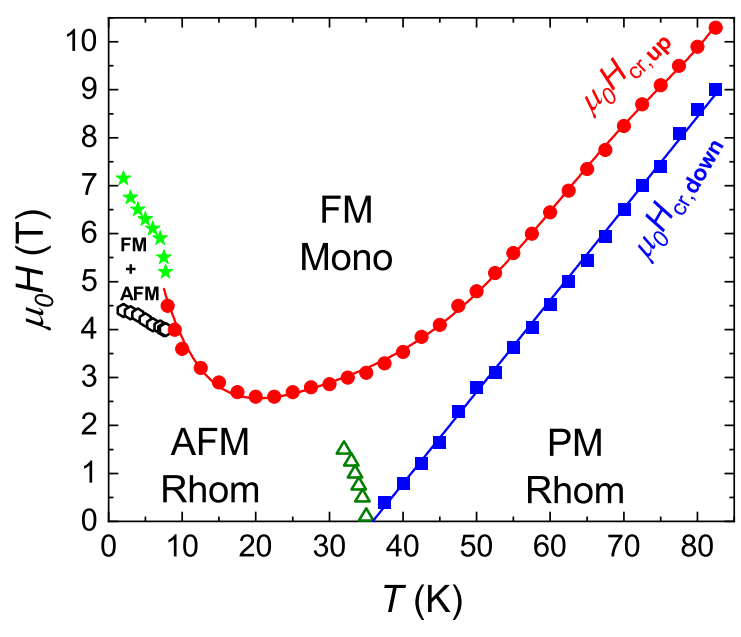

FIG. 14. Magnetic and crystallographic phase diagram of $\mathrm{La}_{0.9} \mathrm{Ce}_{0.1} \mathrm{Fe}_{12} \mathrm{~B}_{6}$. AFM, PM, and FM label different magnetic phases, and Rhom and Mono denote different crystallographic structures, as defined throughout the text. The critical field for the upward ( $\left.\mu_{0} H_{\mathrm{cr}, \text { up }}\right)$ and downward $\left(\mu_{0} H_{\mathrm{cr} \text {,down }}\right)$ field variation as a function of temperature. The critical field has been derived from the maximum of the field derivative of the magnetization isotherms. The open hexagons and closed stars stand for the transition field of the first- and second-step transitions, respectively. The open triangles represent the Néel temperature, $T_{\mathrm{N}}$.

exhibits the inverse trend at temperatures exceeding $25 \mathrm{~K}$. Below $25 \mathrm{~K}$, the critical field of the AFM-FM transition increases upon cooling because of the enhancement of the negative exchange interactions and the reduction of the thermal fluctuations of the moments and elasticity of the crystal structure in the AFM phase [17,20,51]. This results in the increase of both the free-energy difference between the two magnetically ordered AFM and FM states, and the critical magnetic field required to complete the metamagnetic transition from one phase to another. In the paramagnetic temperature region, $\mu_{0} H_{\text {cr,up }}$ evolves proportionally to the square of temperature $T^{2}$ ( $T^{2}$ dependence) at low temperatures and presents a $T$ dependence on the high-temperature side, in agreement with the spin-fluctuation theory for itinerant-electron metamagnetic systems [52-54]. It is worth to notice that the metamagnetic transition in $\mathrm{La}_{0.9} \mathrm{Ce}_{0.1} \mathrm{Fe}_{12} \mathrm{~B}_{6}$ extends over a wide temperature range including below and well beyond $T_{\mathrm{N}}$. The Néel temperature $T_{\mathrm{N}}$ (AFM-PM second-order phase transition) is weakly sensitive to the applied magnetic field. $T_{\mathrm{N}}$ slightly shifts to lower temperatures with increasing external field. The characteristic features of this phase diagram are large hysteresis associated with the magnetostructural transition in the magnetic data and a negative shift of the Néel temperature with applied magnetic field.

In addition to the three distinct magnetic phase regionsAFM, FM, and PM-another salient feature of the present phase diagram is the very low-temperature region, where the intermetallic compound $\mathrm{La}_{0.9} \mathrm{Ce}_{0.1} \mathrm{Fe}_{12} \mathrm{~B}_{6}$ may be in an AFM, phase mixture AFM+FM, or FM state depending on the thermal and magnetic history of the sample. For instance, when the sample is cooled in zero magnetic field from room temperature to $2 \mathrm{~K}$ and afterward magnetized by the application 
of a 4-T field, it will stay in the pure AFM ground state at $2 \mathrm{~K}$ and $4 \mathrm{~T}$. When cooled from high temperatures down to $2 \mathrm{~K}$ in a 4-T magnetic field, the system will cross the onset AFM-FM phase boundary and undergo a partial AFM $\rightarrow$ FM order-order magnetic transformation; in such conditions the compound will remain in the phase mixture (or phasesegregated) AFM+FM state.

At this point, the issue of the origin of the ultrasharp magnetization jumps, also seen in the magnetostriction data, must be taken into consideration. It is quite unambiguous that these unusual and anomalous features observed in $\mathrm{La}_{0.9} \mathrm{Ce}_{0.1} \mathrm{Fe}_{12} \mathrm{~B}_{6}$ are not solely magnetic in origin. Our experimental findings undoubtedly prove that they possess a contribution from the strong spin-lattice coupling. Several interpretations have been suggested for the avalanchelike transitions phenomenon in intermetallic compounds and phase-separated oxides (manganese-based perovskites); however, a scenario based on the martensiticlike transition triggered by the external magnetic field appears to be the most prominent one [55-57].

Let us depict the generation of field-induced staircaselike transitions in the magnetization process within the scope of such a martensiticlike transformation. The magnetic ground state of $\mathrm{La}_{0.9} \mathrm{Ce}_{0.1} \mathrm{Fe}_{12} \mathrm{~B}_{6}$ is AFM with a rhombohedral crystallographic structure. When a suitable magnetic field is applied, the FM phase (monoclinic) begins to grow inside the AFM matrix. The field-induced structural distortion (martensiticlike transformation) between the monoclinic and rhombohedral unit cells produces elastic strains at the AFM/FM interfaces. With the application of magnetic field, FM regions are likely to develop but the interfacial (martensitic) constraints obstruct the growth of the FM domains. As the external field is progressively raised, the driving force acting on the spins raises as well. When the magnetic force is strong enough to prevail over the elastic strain energy, the FM component grows catastrophically, giving rise to extremely sharp steps. These abrupt jumps can be considered as a burstlike development of the FM component within the AFM matrix. The magnetic field-induced lattice distortion which is driven by magnetoelastic coupling is likely responsible for the steplike metamagnetic transitions in this compound.
Similar sharp jumps and staircaselike transitions have been recently observed in other metamagnets such as FeRh-based systems [58-61]. Some authors attributed their origin to the AFM-FM phase boundary motion that is pinned by defects or inhibited by the stray field of the portions that have already converted [58]; others proposed the combined effects of the transition hysteresis and the temperature dependence of the order parameter [59]. Instead, Uhlîr et al. [61] suggested an alternative mechanism driven by the stronger exchange correlations of the long-range FM order compared to the AFM state, i.e., the robustness of the FM exchange to local strain and disorder when compared with the AFM exchange. This should lead to residual FM domains at low temperatures that serve as seeds for the first-order AFM to FM metamagnetic phase transition.

\section{SUMMARY AND CONCLUSIONS}

In summary, we have carried out a detailed investigation of the magnetic, magnetoelastic, and structural properties of $\mathrm{La}_{0.9} \mathrm{Ce}_{0.1} \mathrm{Fe}_{12} \mathrm{~B}_{6}$. We discovered that the first-order AFMFM transition occurs simultaneously with a crystallographic transition from a rhombohedral to a monoclinic structure. This field-induced symmetry-lowering structural distortion is driven by magnetoelastic effects. The FM order sets in the monoclinic crystal structure which can be described in $C 2 / m$ symmetry group, a subgroup of $R \overline{3} m$, the space group of the rhombohedral $\mathrm{SrNi}_{12} \mathrm{~B}_{6}$-type crystallographic structure adopted by the AFM and PM phases. A huge volume magnetostriction, $\Delta V / V=1.15 \%$, was observed across the magnetic field-induced AFM-FM metamagnetic transition, making this intermetallic compound a potential candidate for magnetostrictive materials. Our experimental findings constitute direct evidence of the strong coupling between magnetic and crystallographic degrees of freedom in $\mathrm{La}_{0.9} \mathrm{Ce}_{0.1} \mathrm{Fe}_{12} \mathrm{~B}_{6}$ system.

In order to establish the magnetic structure of the FM (monoclinic) phase and gain a deeper insight into the coupling between crystal lattice and magnetism in this intermetallic compound, neutron diffraction experiments in applied magnetic fields and further theoretical investigations and electronic band-structure calculations are required.
[1] J. Mira, F. Rivadulla, J. Rivas, A. Fondado, T. Guidi, R. Caciuffo, F. Carsughi, P. G. Radaelli, and J. B. Goodenough, Phys. Rev. Lett. 90, 097203 (2003).

[2] A. Zieba, Y. Shapira, and S. Foner, Phys. Lett. A 91, 243 (1982).

[3] C. P. Bean and D. S. Rodbell, Phys. Rev. 126, 104 (1962).

[4] A. Asamitsu, Y. Moritomo, Y. Tomioka, T. Arima, and Y. Tokura, Nature (London) 373, 407 (1995).

[5] E. M. Levin, V. K. Pecharsky, K. A. Gschneidner, Jr., and G. J. Miller, Phys. Rev. B 64, 235103 (2001).

[6] C. Magen, L. Morellon, P. A. Algarabel, C. Marquina, and M. R. Ibarra, J. Phys.: Condens. Matter 15, 2389 (2003).

[7] S. B. Roy, M. K. Chattopadhyay, P. Chaddah, J. D. Moore, G. K. Perkins, L. F. Cohen, K. A. Gschneidner, Jr., and V. K. Pecharsky, Phys. Rev. B 74, 012403 (2006).
[8] V. K. Pecharsky, A. P. Holm, K. A. Gschneidner, Jr., and R Rink, Phys. Rev. Lett. 91, 197204 (2003).

[9] V. K. Pecharsky and K. A. Gschneidner, Jr., Adv. Mater. 13, 683 (2001).

[10] L. Morellon, P. A. Algarabel, M. R. Ibarra, J. Blasco, B. GarcíaLanda, Z. Arnold, and F. Albertini, Phys. Rev. B 58, R14721 (1998).

[11] V. K. Pecharsky and K. A. Gschneidner, Jr., Phys. Rev. Lett. 78, 4494 (1997).

[12] A. Haldar, N. K. Singh, Ya. Mudryk, K. G. Suresh, A. K. Nigam, and V. K. Pecharsky, Solid State Commun. 150, 879 (2010).

[13] H. Yibole, A. K. Pathak, Y. Mudryk, F. Guillou, N. Zarkevich, S. Gupta, V. Balema, and V. K. Pecharsky, Acta Mater. 154, 365 (2018).

[14] M. Fallot and R. Hocart, Rev. Sci. 77, 498 (1939). 
[15] G. Shirane, R. Nathans, and C. W. Chen, Phys. Rev. 134, A1547 (1964).

[16] J. A. Arregi, O. Caha, and V. Uhlîr, Phys. Rev. B 101, 174413 (2020).

[17] L. V. B. Diop, O. Isnard, and J. Rodríguez-Carvajal, Phys. Rev. B 93, 014440 (2016).

[18] S. Fujieda, K. Fukamichi, and S. Suzuki, J. Magn. Magn. Mater. 421, 403 (2017).

[19] L. V. B. Diop and O. Isnard, Appl. Phys. Lett. 108, 132401 (2016).

[20] L. V. B. Diop and O. Isnard, Phys. Rev. B 97, 014436 (2018).

[21] L. V. B. Diop and O. Isnard, J. Appl. Phys. 119, 213904 (2016).

[22] L. V. B. Diop, O. Isnard, Z. Arnold, J. P. Itié, J. Kastil, and J. Kamarad, Solid State Commun. 252, 29 (2017).

[23] G. I. Miletic and Z. Blazina, J. Magn. Magn. Mater. 323, 2340 (2011).

[24] G. I. Miletic and Z. Blazina, J. Alloys Compd. 430, 9 (2007).

[25] M. Rosenberg, T. Sinnemann, M. Mittag, and K. H. J. Buschow, J. Alloys Compd. 182, 145 (1992).

[26] Q. A. Li, C. H. de Groot, F. R. de Boer, and K. H. J. Buschow, J. Alloys Compd. 256, 82 (1997).

[27] K. H. J. Buschow, D. B. de Mooij, and H. M. van Noort, J. Less-Common Met. 125, 135 (1986).

[28] M. Mittag, M. Rosenberg, and K. H. J. Buschow, J. Magn. Magn. Mater. 82, 109 (1989).

[29] K. Niihara and S. Yajima, Chem. Lett. 1, 875 (1972).

[30] Y. B. Kuz'ma, G. V. Chernyak, and N. F. Chaban, Dopov. Akad. Nauk. Ukr. RSR Ser. A 12, 80 (1981).

[31] W. Jung and D. Quentmeier, Z. Kristallogr. 151, 121 (1980).

[32] F. Mesquita, S. G. Magalhaes, P. Pureur, L. V. B. Diop, and O. Isnard, Phys. Rev. B 101, 224414 (2020).

[33] A. Barlet, J. C. Genna, and P. Lethuillier, Cryogenics 31, 801 (1991).

[34] T. Faske and W. Donner, J. Appl. Crystallogr. 51, 761 (2018).

[35] J. Rodriguez-Carvajal, Physica B 192, 55 (1993).

[36] R. Huang, Y. Liu, W. Fan, J. Tan, F. Xiao, L. Qian, and L. Li, J. Am. Chem. Soc. 135, 11469 (2013).

[37] A. W. Sleight, Inorg. Chem. 37, 2854 (1998).

[38] K. Irisawa, A. Fujita, K. Fukamichi, M. Yamada, H. Mitamura, T. Goto, and K. Koyama, Phys. Rev. B 70, 214405 (2004).

[39] A. Fujita, Y. Akamatsu, and K. Fukamichi, J. Appl. Phys. 85, 4756 (1999).

[40] L. Morellon, J. Blasco, P. A. Algarabel, and M. R. Ibarra, Phys. Rev. B 62, 1022 (2000).

[41] Ya. Mudryk, A. P. Holm, K. A. Gschneidner, Jr., and V. K. Pecharsky, Phys. Rev. B 72, 064442 (2005).

[42] Ya. Mudryk, D. Paudyal, V. K. Pecharsky, and K. A. Gschneidner, Jr., Phys. Rev. B 77, 024408 (2008).

[43] F. Ishikawa, K. Koyama, K. Watanabe, and H. Wada, Jpn. J. Appl. Phys. 42, L918 (2003).
[44] A. I. Kurbakov, V. A. Ryzhov, V. V. Runov, E. O. Bykov, I. I. Larionov, V. V. Deriglazov, C. Martin, and A. Maignan, Phys. Rev. B 100, 184424 (2019).

[45] J. B. Goodenough, in Handbook on the Physics and Chemistry of Rare Earths, edited by K. A. Gschneidner, Jr., J. C. Bünzli, and V. K. Pecharsky (Elsevier, New York, 2003), Vol. 33, Chap. 214, p. 249.

[46] Y. Qiao, Y. Song, K. Lin, X. Liu, A. Franz, Y. Ren, J. Deng, R. Huang, L. Li, J. Chen, and X. Xing, Inorg. Chem. 58, 5380 (2019).

[47] L. V. B. Diop, O. Isnard, M. Amara, F. Gay, and J. P. Itié, J. Alloys Compd. 845, 156310 (2020).

[48] B. Li, X. H. Luo, H. Wang, W. J. Ren, S. Yano, C. W. Wang, J. S. Gardner, K. D. Liss, P. Miao, S. H. Lee, T. Kamiyama, R. Q. Wu, Y. Kawakita, and Z. D. Zhang, Phys. Rev. B 93, 224405 (2016).

[49] J. Lin, P. Tong, K. Zhang, H. Tong, X. Guo, C. Yang, Y. Wu, M. Wang, S. Lin, L. Chen, W. Song, and Y. Sun, Appl. Phys. Lett. 109, 241903 (2016).

[50] M. Azuma, W. Chen, H. Seki, M. Czapski, S. Olga, K. Oka, M. Mizumaki, T. Watanuki, N. Ishimatsu, N. Kawamura, S. Ishiwata, M. G. Tucker, Y. Shimakawa, and J. P. Attfield, Nature Commun. 2, 347 (2011).

[51] E. M. Levin, K. A. Gschneidner, Jr., and V. K. Pecharsky, Phys. Rev. B 65, 214427 (2002).

[52] T. Moriya, Spin Fluctuations in Itinerant Electron Magnetism (Springer-Verlag, Berlin, 1985).

[53] H. Yamada and T. Goto, Phys. Rev. B 68, 184417 (2003).

[54] T. Goto, K. Fukamichi, and H. Yamada, Physica B 300, 167 (2001).

[55] V. Hardy, S. Majumdar, S. J. Crowe, M. R. Lees, D. Mck. Paul, L. Hervé, A. Maignan, S. Hébert, C. Martin, C. Yaicle, M. Hervieu, and B. Raveau, Phys. Rev. B 69, 020407(R) (2004).

[56] B. Maji, K. G. Suresh, and A. K. Nigam, Europhys. Lett. 91, 37007 (2010).

[57] A. Haldar, K. G. Suresh, and A. K. Nigam, Phys. Rev. B 78, 144429 (2008).

[58] A. M. Chirkova, K. P. Skokov, Y. Skourski, F. Scheibel, A. Y. Karpenkov, A. S. Volegov, N. V. Baranov, K. Nielsch, L. Schultz, K.-H. Müller, T. G. Woodcock, and O. Gutfleisch, Phys. Rev. Materials 5, 064412 (2021).

[59] J. del Valle, N. Ghazikhanian, Y. Kalcheim, J. Trastoy, M.-H. Lee, M. J. Rozenberg, and I. K. Schuller, Phys. Rev. B 98, 045123 (2018).

[60] J. A. Arregi, M. Horký, K. Fabianová, R. Tolley, E. E. Fullerton, and V. Uhlîr, J. Phys. D: Appl. Phys. 51, 105001 (2018).

[61] V. Uhlîr, J. A. Arregi1, and E. E. Fullerton, Nat. Commun. 7, 13113 (2016). 